ARTICLE

https://doi.org/10.1038/s41467-019-09482-6

\title{
The IncRNA Neat1 promotes activation of inflammasomes in macrophages
}

\author{
Pengfei Zhang ${ }^{1}$, Limian Cao ${ }^{1}$, Rongbin Zhou (D) ${ }^{1}$, Xiaolu Yang ${ }^{2} \&$ Mian Wu ${ }^{1,3}$
}

The inflammasome has an essential function in innate immune, responding to a wide variety of stimuli. Here we show that the IncRNA Neat1 promotes the activation of several inflammasomes. Neat1 associates with the NLRP3, NLRC4, and AIM2 inflammasomes in mouse macrophages to enhance their assembly and subsequent pro-caspase-1 processing. Neat 1 also stabilizes the mature caspase- 1 to promote interleukin-1 $\beta$ production and pyroptosis. Upon stimulation with inflammasome-activating signals, Neat1, which normally resides in the paraspeckles, disassociates from these nuclear bodies and translocates to the cytoplasm to modulate inflammasome activation using above mechanism. Neat 1 is also up-regulated under hypoxic conditions in a HIF- $2 \alpha$-dependent manner, mediating the effect of hypoxia on inflammasomes. Moreover, in the mouse models of peritonitis and pneumonia, Neat1 deficiency significantly reduces inflammatory responses. These results reveal a previously unrecognized role of IncRNAs in innate immunity, and suggest that Neat1 is a common mediator for inflammasome stimuli.

\footnotetext{
${ }^{1}$ The Chinese Academy of Sciences (CAS) Key Laboratory of Innate Immunity and Chronic Disease, CAS Center for Excellence in Cell and Molecular Biology, School of Life Sciences, University of Science and Technology of China, 230026 Hefei, China. ${ }^{2}$ Department of Cancer Biology and Abramson Family Cancer Research Institute, Perelman School of Medicine, University of Pennsylvania, Philadelphia, PA 19104, USA. ${ }^{3}$ Translational Research Institute, Henan Provincial People's Hospital, Academy of Medical Science, Zhengzhou University, Zhengzhou, Henan Key Laboratory of Stem Cell Differentiation and Modification, School of Clinical Medicine, Henan University, 450003 Zhengzhou, China. These authors contributed equally: Pengfei Zhang, Limian Cao. Correspondence and requests for materials should be addressed to X.Y. (email: xyang@mail.med.upenn.edu) or to M.W. (email: wumian@ustc.edu.cn)
} 
nflammasomes are a group of multicomponent signaling platforms in the cytoplasm that control inflammatory response and anti-pathogen defense against a wide range of infection and damage signals ${ }^{1-4}$. These signals, including pathogen-associated molecular patterns (PAMPs) and damageassociated molecular patterns (DAMPs) ${ }^{2,5}$, directly or indirectly activate one or more innate pattern recognition receptors (PRRs), which include nucleotide-binding domain (NBD) and leucinerich repeat (LRR)-containing receptors (NLRs, e.g., NLRP1, NLRP3, and NLRC4), cytosolic DNA sensors (AIM2), and Pyrin (also known as TRIM20) ${ }^{6-8}$. Upon activation, the sensor proteins bind to and induce the oligomerization of a common adaptor protein, apoptosis-associated speck-like protein containing CARD (ASC), leading to the formation of a single macromolecular aggregate known as ASC speck $^{9-11}$. Oligomerized ASC recruits pro-caspase- $1^{12}$, facilitating its auto-processing into the mature subunits ${ }^{13}$. Active caspase- 1 mediates proteolytic maturation of pro-inflammatory cytokines interleukin $1 \beta$ (IL-1 $\beta$ ) and IL-18 and elicits pyroptosis, a form of programmed cell death that exhibits features of both apoptosis (e.g., DNA fragmentation) and necrosis (e.g., plasma membrane rupture) $)^{2,4,14-16}$.

While adequate inflammasome activation is crucial for the elimination of pathogens and damaged cells ${ }^{17,18}$, dysregulation of inflammasome contributes to autoimmune, cancer, neurodegenerative disorders, and other diseases ${ }^{19}$. Nevertheless, the regulation of inflammasomes is not well understood. Several inflammasomes respond to a limited set of signals. For example, the AIM2 and NLRC4 inflammasomes are assembled upon the sensing of double-stranded DNA (dsDNA) and specific bacterial proteins, respectively ${ }^{20,21}$, while the inflammasome formed with NLRP1 or its murine homolog Nlrp1b is activated by anthrax lethal toxin (LeTx) and 2-deoxy-D-Glucose (2DG) ${ }^{22,23}$. In contrast, the NLRP3 inflammasome is activated by an extraordinarily diverse array of PAMPs including several viral, bacterial, fungal pathogens, and DAMPs, such as crystalline, particulate (e.g., uric acid crystals, asbestos, and alum), extracellular ATP, poreforming toxins, as well as change in cellular environment, notably hypoxia $5,24,25$. A salient unresolved issue is how various inflammasomes collectively are able to respond to such a wide spectrum of stimuli.

The majority of transcripts transcribed from human or mouse genome are non-coding RNAs ${ }^{26,27}$. Many are long non-coding RNAs (lncRNAs), which are defined as transcripts longer than 200 nucleotides but lacking significant protein coding capacity ${ }^{28}$. Thousands of lncRNAs have been identified to date $26,29-31$, yet only a small fraction of them are characterized. In the context of innate immunity, while a few lncRNAs have been implicated in regulation of inflammasome, including LincRNA-Gm4419, LincRNA-Cox2, and lincRNA-EPS ${ }^{32-34}$, none have been reported to directly involve in inflammasome assembly.

In the current study, we examine lncRNAs that associate with NLRP3 inflammasome in mouse macrophages and identify Neat 1 (nuclear enriched abundant transcript 1), a lncRNA transcribed from the multiple endocrine neoplasia locus (hence also known as Men). Neat1 and its human ortholog NEAT1 maintain the structural integrity of the paraspeckles ${ }^{35}$, a specific type of nuclear bodies in the interchromatin space whose function remains poorly understood ${ }^{36}$. NEAT1 also regulates the expression of a group of chemokines and cytokines, including IL-6 and CXCL10, through the MAPK pathway ${ }^{37}$. Of note, the expression of Neat 1 is stimulated by many stimuli that also activate inflammasome, including infection of various viruses and some intracellular damages (e.g., ROS) that stabilize hypoxia-inducible factors (HIFs) and the tumor suppressor $\mathrm{p} 53^{38-40}$.

Here we find that Neat1 promotes the activation of NLRP3, NLRC4, and AIM2 inflammasomes and enhances caspase-1 activation, cytokine production, and pyroptotic cell death. Mechanistically, Neat1 binds to pro-caspase-1 and facilitates the assembly of inflammasomes, and also stabilizes the mature caspase- 1 and increases caspase-1protease activity. In response to various inflammasome-activating signals, Neat 1 is released from paraspeckles and translocated to the cytoplasm to participate in inflammasome activity. Our findings establish a direct role for lncRNAs in regulating inflammasomes and suggest that Neat 1 may represent a downstream convergence point for inflammasome stimuli.

\section{Results}

Neat1 enhances the activation of the NLRP3 inflammasome. To investigate whether lncRNAs may regulate inflammasomes, we set out to identify lncRNAs that are associated with the NLRP3 inflammasome in murine immortalized bone marrow-derived macrophages (iBMDMs). We primed iBMDMs with lipopolysaccharides (LPS) and subsequently treated them with the potassium ionophore nigericin to activate the NLRP3 inflammasome. After treating these cells with UV to stabilize proteinRNA interactions, we immunoprecipitated NLRP3 inflammasomes using anti-NLRP3 antibody and analyzed the associated RNAs by high-throughput sequencing (GSE118722). Several noncoding RNAs were found to be enriched in NLRP3 inflammasomes, including those encoded by Ankrd52, Hipk3, Pou2f2, Neat1, Gm26917, Gm42418, Snord34, Junos, Gm26767, and Gm23935 (Supplementary Fig. 1a). Among them were four lncRNAs: Gm26917, Gm42418, Gm26767, and Neat1 (Supplementary Fig. 1a).

To evaluate their effect on the NLRP3 inflammasome, we stably knocked down each of these four lncRNAs in iBMDMs using short hairpin RNA (shRNA). Knocking down Gm26917, Gm42418, or Gm26767 lncRNAs had no effect on the NLRP3 inflammasome (data not shown). Neat1 (Gene ID: 66961), is expressed in two isoforms, $3.2 \mathrm{~kb}$ Neat1_1 (NR_003513.3, also known as Men epsilon) and $20 \mathrm{~kb}$ Neat1_2 (NR_003513.3, also known as Men beta) (Supplementary Fig. 1b). We knocked down Neat1_1 and Neat1_2 simultaneously using two independent shRNAs (\#1 and \#2; Supplementary Fig. 1b, c). Interestingly, the knockdown of total Neat1 resulted in a strong reduction in oligomerization of ASC, indicative of impaired assembly of the inflammasome (Fig. 1a). This was accompanied by a noticeable reduction in the activation of caspase-1, processing of prointerleukin $1 \beta$ (pro-IL-1 $\beta$ ) (Fig. 1a), and secretion of mature IL$1 \beta$ (Fig. 1b, c). We also knocked down Neat1_2 alone with an isoform-specific shRNA (Supplementary Fig. 1b, d), and observed an almost indistinguishable set of results (Fig. 1d, e). Conversely, we forced the expression of Neat1. Neat1_2 is too long to be readily cloned and expressed. Therefore, we used the short variant Neat1_1 (Supplementary Fig. 1b). When Neat1_1 was overexpressed in iBMDMs (Supplementary Fig. 1h), LPS/nigericininduced ASC aggregation, pro-caspase- 1 cleavage, and IL- $1 \beta$ secretion were all increased (Figs. 1f, g).

Knocking down total Neat1 or Neat1_2 alone, or overexpressing Neat1_1, did not alter the expression of NLRP3, ASC, pro-caspase-1, or pro-IL-1 $\beta$ (Fig. 1a, d, f and Supplementary Fig. 1h), suggesting that Neat1 promotes the assembly of the NLRP3 inflammasome rather than increasing the expression of its components or downstream targets. These changes in Neat 1 levels did not influence the secretion of TNF- $\alpha$ either (Supplementary Fig. 1e-g, i), indicating a specific effect of Neat1 on NLRP3 inflammasome-mediated cytokine production.

To verify the stimulatory effect of Neat1 on the NLRP3 inflammasome, we generated Neat1 knockout $\left(\right.$ Neat $\left.1^{-/}\right)$mice on the C57BL/6 background using CRISPR/Cas9-based genome 
a

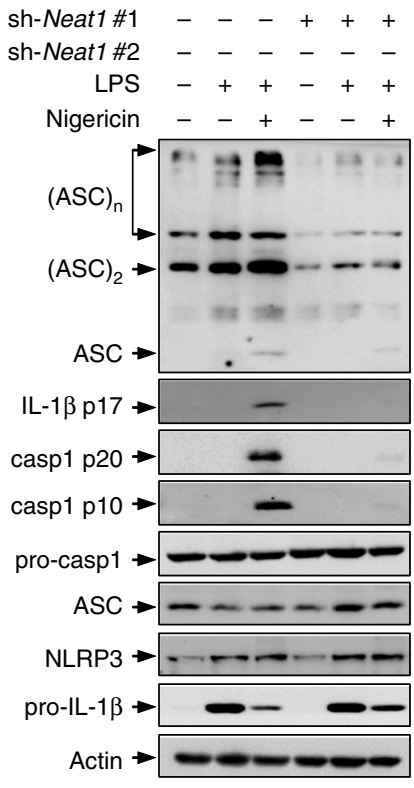

d

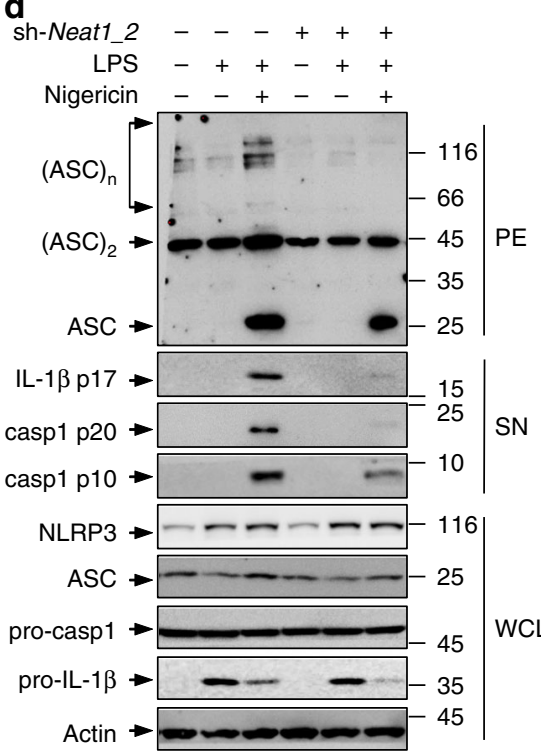

Neat1 1 - --++++

LPS -++-++

Nigericin $-\ldots+-\ldots$

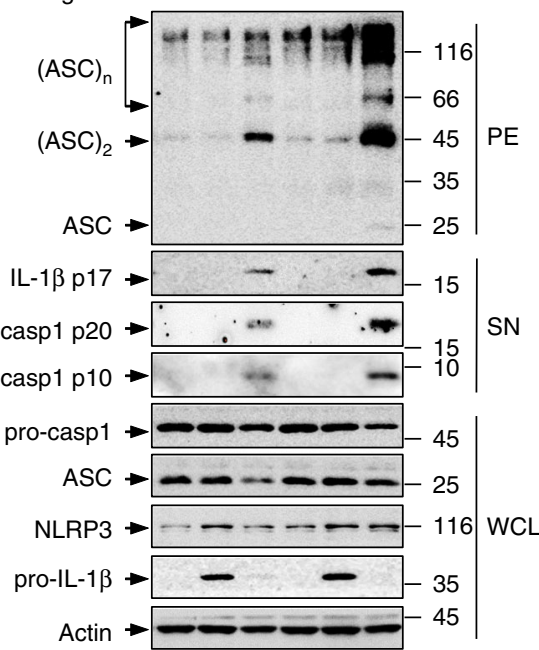

sh-ctrl

sh-Neat1 \#1

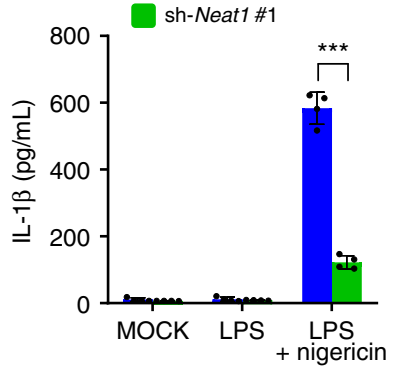

C

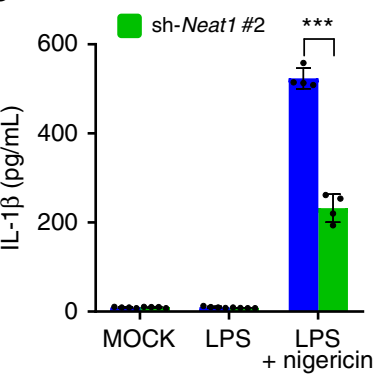

e

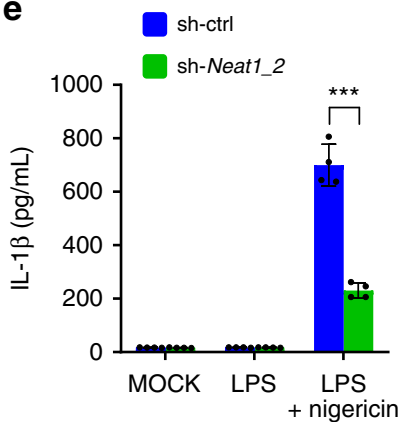

g

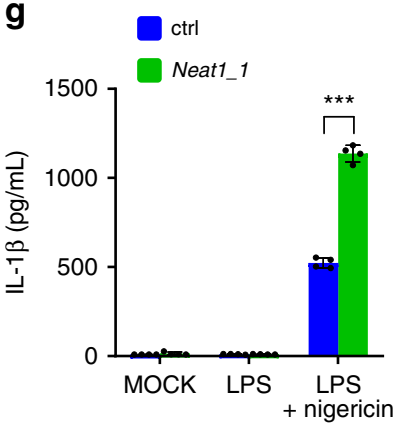

h

$\begin{array}{rrrrrr}\text { Neat }^{1 /+} & + & + & - & - \\ \text { Neat } 1^{-/-} & - & - & + & + & + \\ \text {LPS } & + & + & - & + & + \\ \text { Nigericin } & - & + & - & +\end{array}$

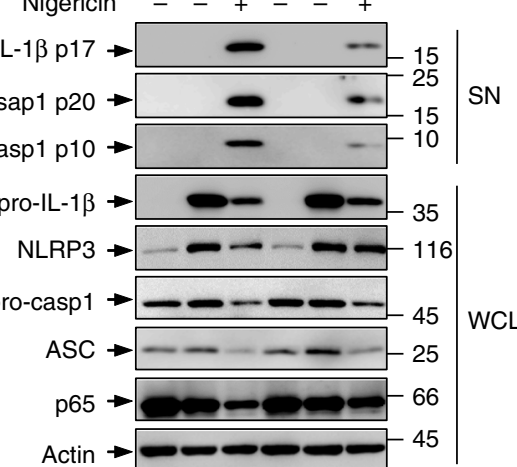

WCL

Fig. 1 Neat1 enhances the activation of the NLRP3 inflammasome. a-c Control iBMDMs and iBMDMs devoid of Neat1 by shRNA \#1 (a, left and b) or \#2 (a, right and $\mathbf{c}$ ) were untreated, primed with LPS, or treated with nigericin after LPS priming. Whole cell lysates (WCL) and the supernatant (SN) and pellet (PE) fractions were analyzed by western blotting for indicated protein levels (a). Culture supernatant was analyzed by ELISA for IL-1 $\beta$ secretion (b, c). $\mathbf{d}-\mathbf{g}$ iBMDMs devoid of Neat1_2 (d, e) or overexpressing Neat1_1 $(\mathbf{f}, \mathbf{g})$ and the corresponding controls were treated with or without LPS or nigericin as indicated, and analyzed for protein levels $(\mathbf{d}, \mathbf{f})$ and IL-1 $\beta$ secretion $(\mathbf{e}, \mathbf{g})$. h, i BMDMs from Neat1 wild-type $\left(\right.$ Neat $\left.1^{+/+}\right)$or knockout $\left(\right.$Neat $\left.1^{-/-}\right)$mice were primed with LPS and then stimulated with nigericin as indicated. Protein levels (h) and IL-1 $\beta$ secretion (i) were analyzed. In $\mathbf{b}, \mathbf{c}, \mathbf{e}, \mathbf{g}$, and $\mathbf{i}$, data shown are mean \pm SD $(n=4) .{ }^{\star \star \star} P<0.001$, two-tailed $t$-test. Source data are provided as a Source Data file 
editing (Supplementary Fig. 1j, k). Upon co-stimulation with nigericin and LPS, BMDMs derived from Neat $1^{-/-}$mice displayed much reduced caspase- 1 activation and IL- $1 \beta$ secretion compared to BMDMs derived from wild-type $\left(\right.$ Neat $\left.1^{+/+}\right)$mice (Fig. 1h, i), despite that both $\mathrm{Neat}^{+/+}$and Neat ${ }^{-/-}$BMDMs contained comparable mRNA and protein levels of NLRP3, ASC, pro-caspase-1, and pro-IL-1 $\beta$ (Fig. $1 \mathrm{~h}$ and Supplementary Fig. $1 \mathrm{k}$ ) and showed similar TNF-a secretion (Supplementary Fig. 11). Moreover, Neat1 $1^{-1-}$ and Neat1 $1^{+/+}$BMDMs also contained comparable levels of RelA/p65 (Fig. 1h and Supplementary Fig. 1k), an upstream activator of the NLRP3 inflammasome, further underscoring the specific effect of Neat1 on the NLRP3 inflammasome.

Consistent with its role in the assembly of the NLRP3 inflammasomes, overexpression of Neat1_1 increased the interactions of NLRP3 with both pro-caspase-1 and ASC (Supplementary Fig. $1 \mathrm{~m}$ ). Therefore, Neat 1 promotes the formation of the NLRP3 inflammasome, leading to enhanced caspase-1 activation and IL-1 $\beta$ maturation.

Neat1 enhances activation of NLRC4 and AIM2 inflammasomes. To evaluate the generality of the effect of Neat 1 , we tested NLRC4 and AIM2 inflammasomes, both of which mediate the activation of caspase-1. Murine macrophages were primed with LPS and then stimulated with flagellin or poly(dA:dT) to active the NLRC4 or AIM2 inflammasome, respectively. iBMDMs devoid of both Neat1_1 and Neat1_2 (Fig. 2a, b), or Neat1_2 alone (Supplementary Fig. 2a, b), showed reduced activation of the NLRC4 and AIM2 inflammasomes, as indicate by a noticeable reduction in caspase- 1 activation and IL-1 $\beta$ processing in response to flagellin and poly(dA:dT) treatment (Fig. 2a, b and Supplementary Fig. 2a, b). Conversely, iBMDMs with forced expression of Neat1_1 displayed augmented activation of both NLRC4 and AIM2 inflammasomes in response to these stimuli (Fig. 2c, d). Moreover, activation of NLRC4 and AIM2 inflammasomes was attenuated in Neat $1^{-1-}$ BMDMs compared to Neat $1^{+/+}$BMDMs (Fig. 2e, f).

In addition, overexpression of Neat1_1 enhanced not only the interactions of ASC with pro-caspase-1 and NLRC4 in flagellinactivated iBMDMs (Fig. 2g), but also the interactions of ASC with pro-caspase-1 and AIM2 in poly(dA:dT)-activated iBMDMs (Fig. 2h). Therefore, Neat1 enhances the assembly and activation of diverse canonical inflammasomes that mediate the activation of caspase-1.

Effect of Neat1 on NLRP3 inflammasome is IL-6-independent. Neat 1 could promote the expression of $\mathrm{IL}-6^{37}$, a proinflammatory cytokine that stimulates immune response. However, in $I L-6^{-1-}$ and $I L-6^{+/+}$BMDMs (Supplementary Fig. 3a) treated with LPS and nigericin, both caspase-1 activation and IL$1 \beta$ secretion occurred to a comparable extent (Fig. 3a and Supplementary Fig. 3b). Moreover, siRNA-mediated knockdown of total Neat1 (Supplementary Fig. 3c, d), or Neat1_2 alone (Supplementary Fig. 3e), similarly inhibited caspase-1 activation and IL- $1 \beta$ secretion in $I L-6^{-I-}$ and $I L-6^{+/+}$BMDMs (Fig. 3b-d). Thus, IL- 6 does not appear to mediate the function of Neat1 on the NLRP3 inflammasome.

Neat1 interacts with caspase-1 p20 domain. Given that Neat1 associates with, and promote the assembly of, the NLRP3 inflammasome in an IL-6-independent manner, we evaluated which component(s) of the NLRP3 inflammasome interacts with Neat1. NLRP3, ASC, and pro-caspase-1 were immunoprecipitated by their specific antibodies from lysates of murine iBMDMs at different stages of stimulation: no stimulation, primed with LPS only, and primed with LPS followed by stimulation with nigericin. Total Neat1 and Neat1_2 were found in pro-caspase-1 immunoprecipitates from cells of all three stages, but in NLRP3 and ASC immunoprecipitates only from cells that are activated by both LPS and nigericin (Fig. $4 \mathrm{a}-\mathrm{c}$ and Supplementary Fig. $4 \mathrm{a}-\mathrm{c}$ ).

In a complementary approach, we pulled down Neat 1 from cell lysates using biotinylated Neat 1 antisense DNA probes, and analyzed the presence of NLRP3 inflammasome components in the pull-down samples. This again revealed that pro-caspase-1 bound to Neat1 in cells of all three stages, whereas ASC and NLRP3 bound to Neat1 only in cells treated with both LPS and nigericin (Fig. 4d and Supplementary Fig. 4d). Thus, Neat1 likely interacts with pro-caspase- 1 constitutively and directly, and with NLRP3 and ASC inducibly and indirectly when these components are incorporated into the inflammasome together with procaspase-1.

Likewise, during the activation of the NLRC4 and AIM2 inflammasomes, Neat 1 bound to pro-caspase-1 directly in cells of all stages: non-stimulated, LPS-primed, and LPS-primed followed by flagellin or poly(dA:dT) stimulation, whereas Neat1 bound to NLRC4/AIM2 and ASC only upon flagellin-dependent NLRC4 inflammasome activation or poly(dA:dT)-dependent AIM2 inflammasome activation (Supplementary Fig. 4e-h).

Upon activation, caspase- 1 is processed to the mature subunits p20 and p10. We observed that more p20 than p10 subunits were pulled down by the Neat1 antisense DNA probes (Fig. 4d). This observation suggested that Neat 1 may directly associate with the p20 subunit of the mature caspase- 1 or the region corresponding to p20 on pro-caspase-1, and indirectly with the p10 subunit within the assembled mature caspase- 1 hetero-tetramer. To verify this, we generated a panel of caspase-1 deletion mutations, each tagged with the Flag epitope (Supplementary Fig. 5a, b). These mutants, as well as full-length pro-caspase-1, were expressing in $293 \mathrm{~T}$ cells, and the cell lysates were incubated with biotin-labeled Neat1_1. This assay showed that Neat1_1 bound to pro-caspase-1 and the p20 region, but not the caspase recruitment domain (CARD) or the p10 region (Fig. 4e). Furthermore, deletion of the p20 region $(\Delta \mathrm{p} 20)$, but not the deletion of the CARD $(\triangle \mathrm{CARD})$ or the p10 region $(\Delta \mathrm{p} 10)$, abolished the interaction with Neat1_1 (Fig. 4f). Therefore, the p20 region of pro-caspase-1 is both sufficient and necessary for the interaction with Neat1, and this interaction persists upon caspase- 1 activation.

To map the caspase-1-binding region on Neat1, we performed NLRP3 CLIP-seq peaks analysis. A strong and specific peak in the 5' region of Neat1_1 and Neat1_2, along with some other weak peaks in the rest of Neat1_1, was identified (Supplementary Fig. 5c), consistent with binding of total Neat1 and Neat1_2 with the NLRP3 inflammasome. We generated three Neat1_1 segments according to the CLIP-seq analysis (Supplementary Fig. 5c), and incubated each of them with HA-tagged procaspase-1. Only full-length Neat1_1 and segment 1, which contained the $5^{\prime} 1 \mathrm{~kb}$ region, could pull down HA-tagged procaspase-1 (Supplementary Fig. 5d), suggesting that Neat1 binds with pro-caspase-1 via its $5^{\prime}$ region.

Neat1 stabilizes the caspase- 1 hetero-tetramers. The active caspase-1, an $\alpha_{2} \beta_{2}$ hetero-tetramer formed by two p10 subunits and two p20 subunits ${ }^{41}$, is turned over by the ubiquitinproteasome pathway ${ }^{42}$. The hetero-tetramer is also unstable and readily dissociates ${ }^{43}$. Neat $1 \_1$ did not affect the ubiquitination of either pro-caspase- 1 or the p20 subunit (Supplementary Fig. 6a). Instead, Neat 1 increased the interaction between p20 and p10 (Fig. 5a, b and Supplementary Fig. 6b, c), suggesting that Neat1 stabilizes the (p20:p10) 2 hetero-tetramer. Mature caspase-1 can also exist as a transient (p33:p10) 2 species, where p33 contains 
a
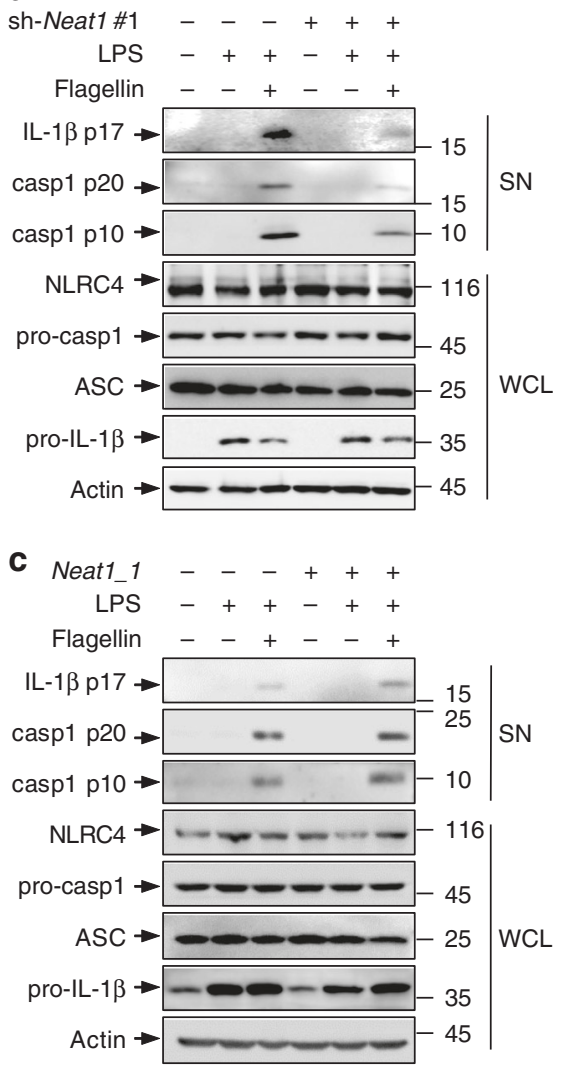

e
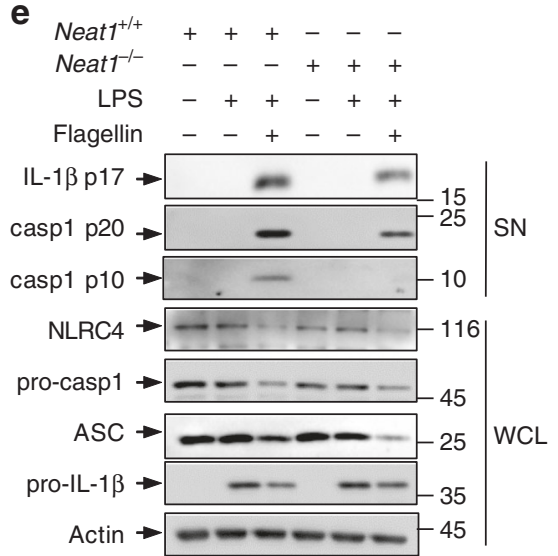

g

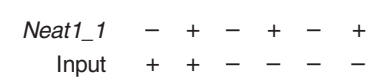

Normal rabbit IgG --++-

Anti-ASC $-\quad-\quad++$

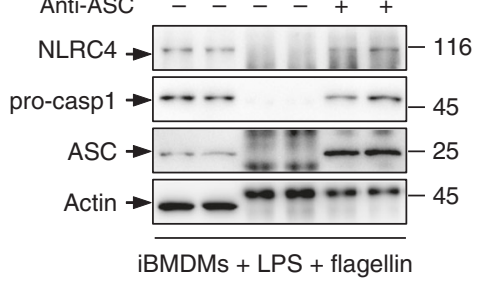

b

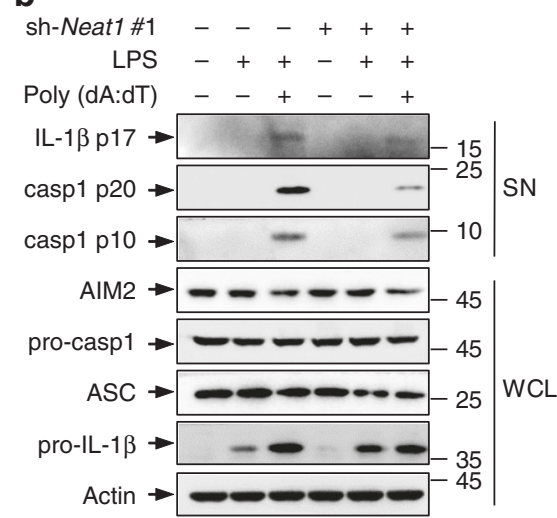

d

d Neat1_1 $--{ }_{-}++$

LPS -++-++

Poly (dA:dT) $\quad \ldots+\ldots$

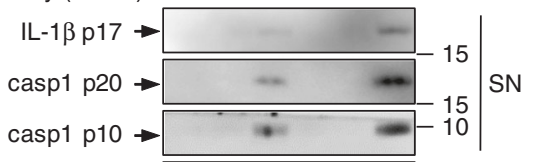

AIM2 $\rightarrow==-45$

pro-casp1 $\rightarrow-45$

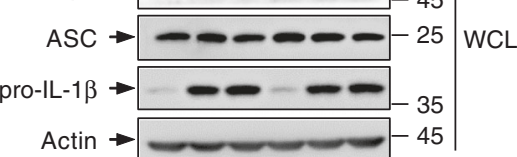

f

f Neat1 $1^{+/+}++---$

Neat1 $1^{-1-}--+++$

LPS -++-++

Poly (dA:dT) $\quad-\quad+\ldots+$

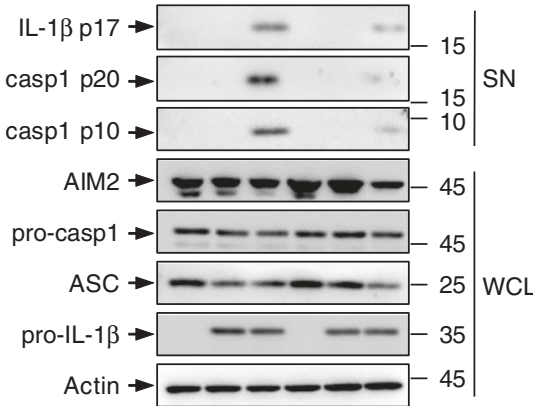

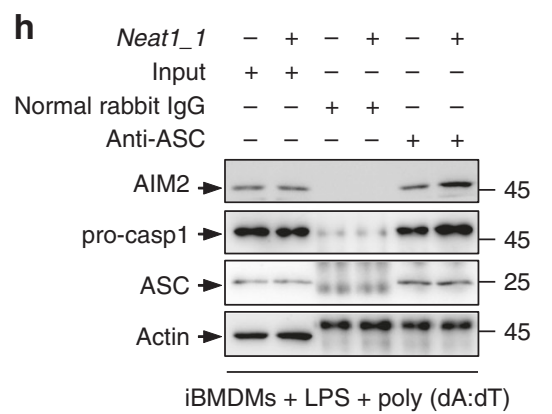

Fig. 2 Neat1 enhances the activation of NLRC4 and AIM2 inflammasomes. a-d iBMDMs devoid of Neat1 by shRNA \#1 (a, b), iBMDMs overexpressing Neat1_1 (c, d), and the corresponding control iBMDMs were primed with LPS, and then stimulated with flagellin (a, c) or poly(dA:dT) (b, d). WCL and SN were analyzed by western blotting. e, $\mathbf{f}$ BMDMs from Neat ${ }^{+/+}$or Neat $1^{-/-}$mice were primed with LPS and then stimulated with flagellin (e) or poly(dA: dT) (f). WCL and SN were analyzed by western blotting. $(\mathbf{g}, \mathbf{h})$ Control iBMDMs and iBMDMs ectopically expressing Neat1_1 were primed with LPS and then stimulated with flagellin $(\mathbf{g})$ or poly $(\mathrm{dA}: \mathrm{dT})(\mathbf{h})$. Cell lysates were immunoprecipitated with normal rabbit IgG or ASC antibody. The samples were analyzed by western blotting as indicated. Source data are provided as a Source Data file 
a

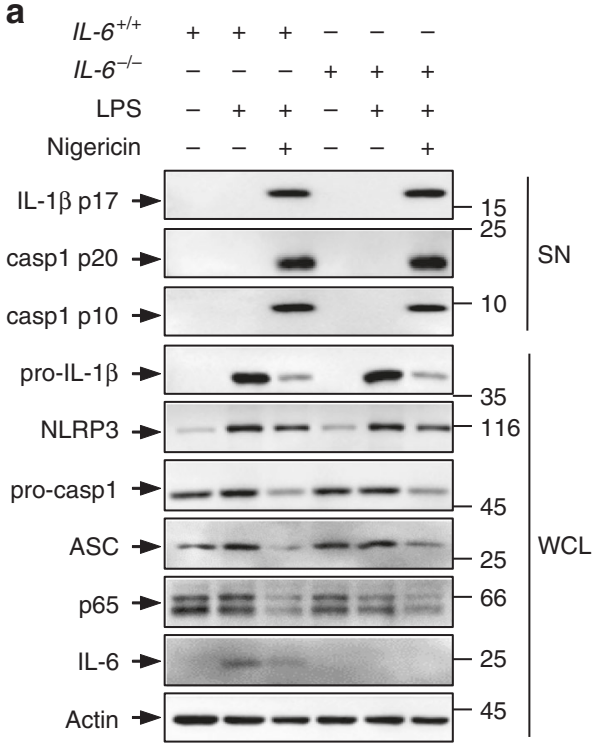

C

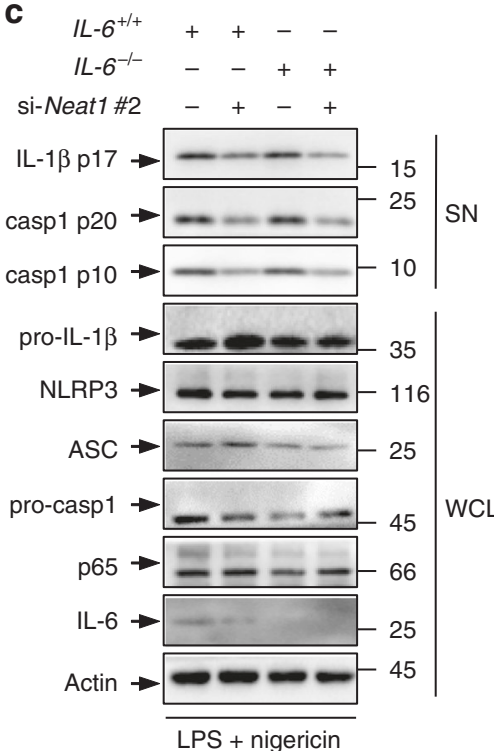

b

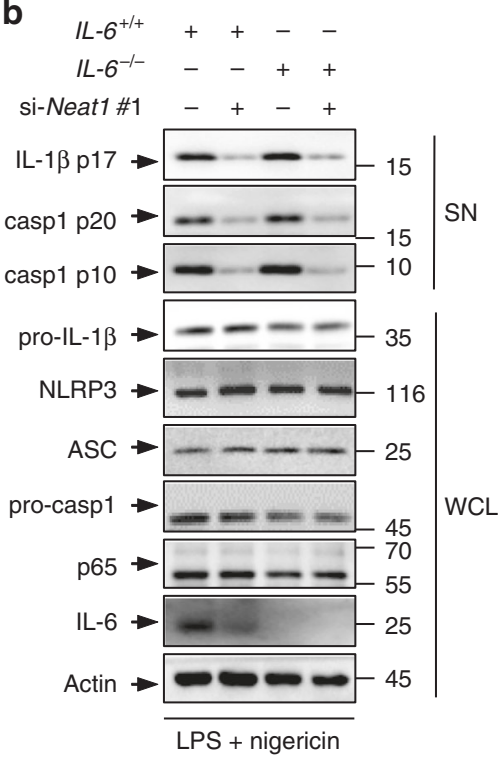

d

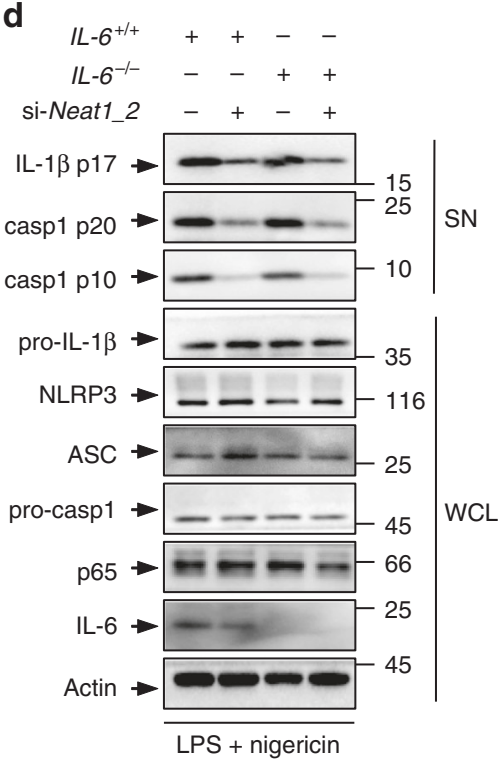

Fig. 3 The effect of Neat1 on the NLRP3 inflammasome activation is independent of IL-6. a BMDMs derived from IL-6 wild-type (IL-6+/+) or knockout (IL-6 $\left.{ }^{-/-}\right)$mice were primed with LPS and then treated with nigericin. Protein levels in total cell lysates and supernatant fraction were analyzed by western blotting. b-d IL-6 $6^{+/+}$or IL-6 $6^{-/-}$BMDMs were transfected with control siRNA (b-d), Neat1 siRNA \#1 (b), Neat1 siRNA \#2 (c), or Neat1_2 siRNA (d). Twenty-four hours later, cells were stimulated with LPS and nigericin. WCL and SN of above cells were subjected to western blotting with indicated antibodies. Source data are provided as a Source Data file

both the CARD and p20 regions ${ }^{44}$. Of note, Neat1_1 could also increase the interaction between p33 and p10 (Fig. $5 c$, d and Supplementary Fig. 5d, e). Therefore, Neat1 stabilizes the subunit-subunit interactions of mature caspase-1 hetero-tetramers. Consistently, caspase- 1 activity was significantly reduced in Neat1 ${ }^{-1-}$ BMDMs compared to Neat1 ${ }^{+/+}$BMDMs upon the formation of NLRP3, NLRC4, or AIM2 inflammasomes (Fig. 5e), while caspase-1 activity was strongly increased in Neat1_1-overexpressing cells compared to control cells upon the formation of these canonical inflammasomes (Fig. 5f). Therefore, Neat1 strengthens the inter-subunit interactions within mature caspase1 and augments the protease activity.

Neat1 promotes caspase-1-dependent pyroptosis. In addition to cytokine secretion, activation of caspase-1 by canonical inflammasomes results in pyroptosis ${ }^{45}$. We observed significant cell death among LPS and nigericin co-stimulated iBMDMs, but not untreated or LPS-primed iBMDMs, as shown by propidium iodide-positive staining and lactate dehydrogenase (LDH) release. Overexpression of Neat1_1 noticeably augmented LPS/nigericininduced pyroptosis (by 120\%; Fig. 6a, b), whereas Neat1 silencing dramatically reduced pyroptosis (by $\sim 80 \%$; Fig. $6 \mathrm{c}$, d). Likewise, Neat1 knockout rendered BMDMs high resistant to pyroptosis (reduced by $\sim 70 \%$; Fig. 6e, f). Therefore, Neat 1 promotes caspase-1-dependent pyroptotic cell death.

Neat1 is exported to cytoplasm upon inflammasome activation. Murine Neat 1 and its human ortholog are highly abundant lncRNAs that are mainly localized to the nucleus of unstressed cells ${ }^{46}$, while assembly of various canonical inflammasomes occurs in the cytoplasm ${ }^{47,48}$. We found that, upon the stimulation of LPS and nigericin, the levels of total Neat1 or the Neat1_2 
a

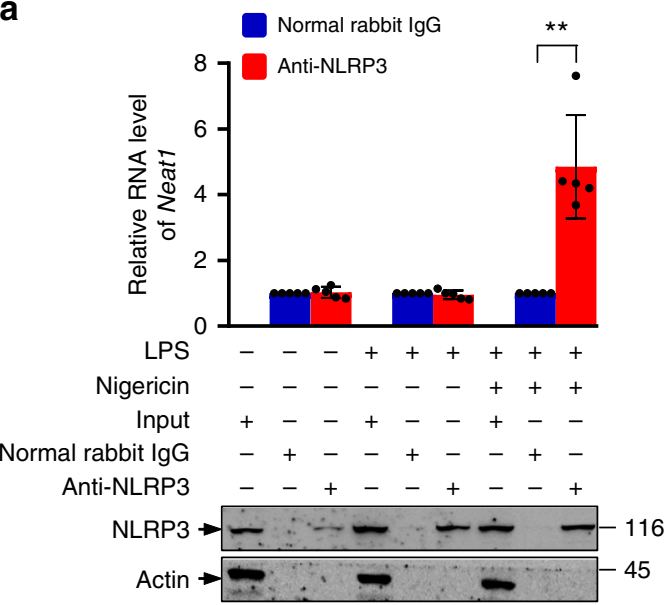

C
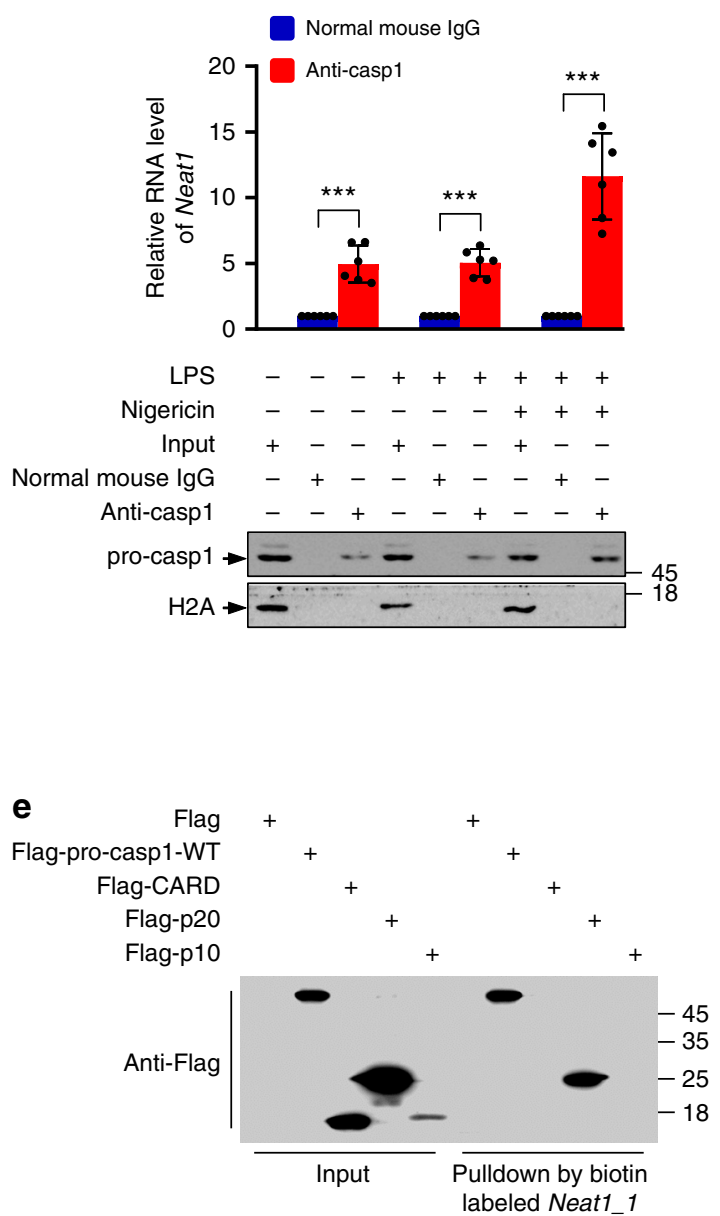

b

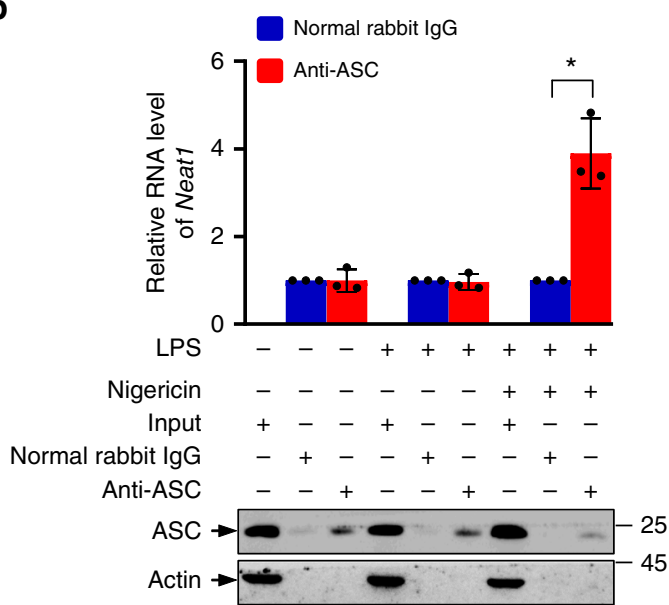

d

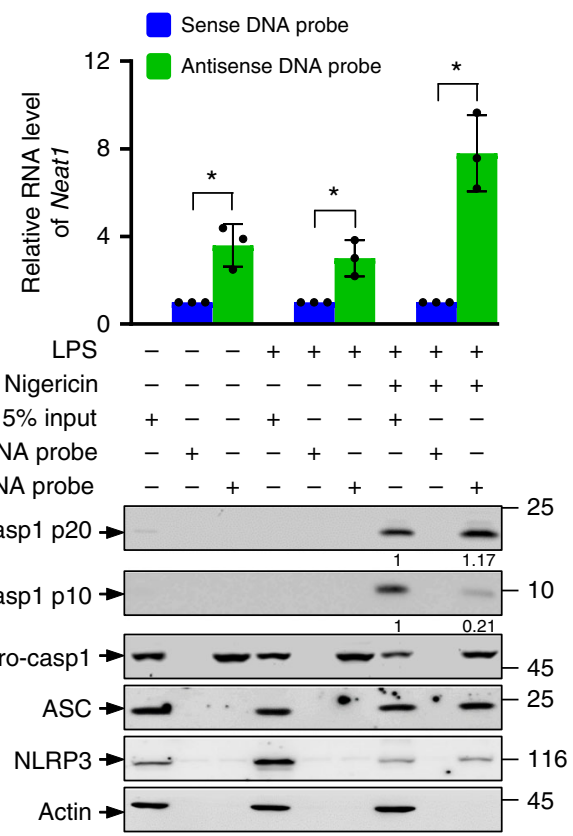

f

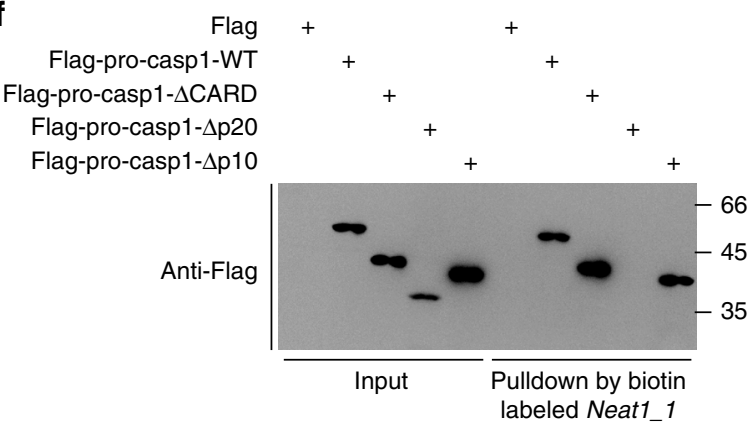

Fig. 4 Neat1 interacts with caspase-1 p20 domain. a-c Cell lysates of normal iBMDMs, LPS-primed iBMDMs or LPS-nigericin-co-stimulated iBMDMs were incubated with normal rabbit IgG (a-c), NLRP3 antibody (a), ASC antibody (b), or caspase-1 antibody (c) for RIP. The immunoprecipitates were analyzed by real-time RT-PCR to exam enrichment efficiency of Neat1 and by Western blotting for protein levels. $\mathbf{d}$ Cell lysates of normal, LPS-primed, or LPSnigericin-co-stimulated iBMDMs were incubated with biotin-labeled Neat1 sense or antisense DNA probes immobilized on beads. The precipitated samples were analyzed by real-time RT-PCR for Neat1 contents, and by western blotting for indicated protein levels. e In vitro transcribed biotin-labeled Neat1_1 was incubated with lysates extracted from 293T cells expressing Flag-tagged wild-type pro-caspase-1, CARD, p20, or p10 for $3 \mathrm{~h}$. The input and biotin pulldown samples were then carried out to western blotting. $\mathbf{f}$ In vitro transcribed biotin-labeled Neat1_1 was incubated with lysates extracted from 293T cells expressing Flag-tagged wild-type pro-caspase- 1 or the indicated deletion mutants for $3 \mathrm{~h}$. The input and biotin pull-down samples were analyzed by western blotting. In $\mathbf{a}$, data shown are mean \pm SD $(n=5)$. In $\mathbf{b}$ and $\mathbf{d}$, data shown are mean $\pm \operatorname{SD}(n=3)$. In $\mathbf{c}$, data shown are mean \pm SD $(n=6)$. ${ }^{\star} P<0.05$, ${ }^{\star \star} P<0.01$, two-tailed $t$-test. Source data are provided as a Source Data file 
a

IB: HA antibody

Neat1_1
Flag-p20
HA-p10
$10 \%$ input

Pulldown by M2 beads

(B)

$-+-+$

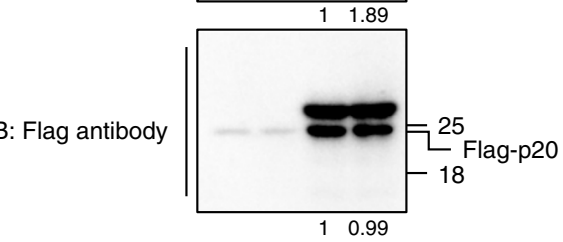

C

IB: HA antibody

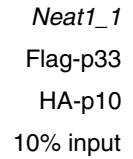

$10 \%$ input

Pulldown by M2 beads

$+$

$\begin{array}{ll}+ & + \\ + & + \\ + & - \\ - & +\end{array}$

IB: Flag antibody
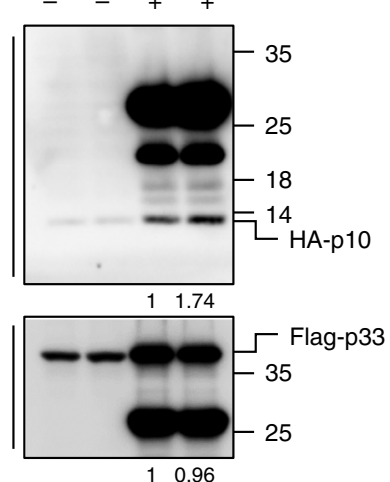

b

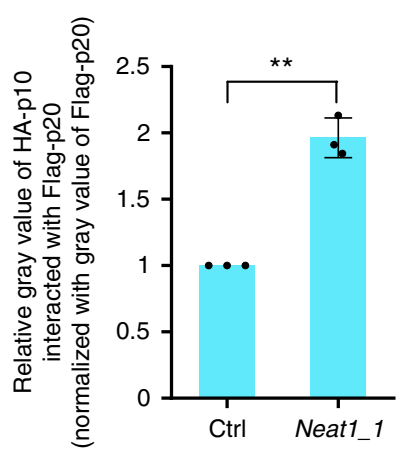

d

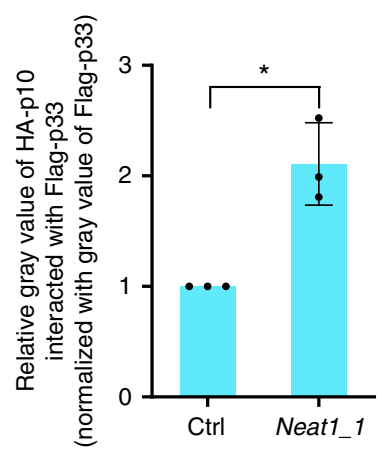

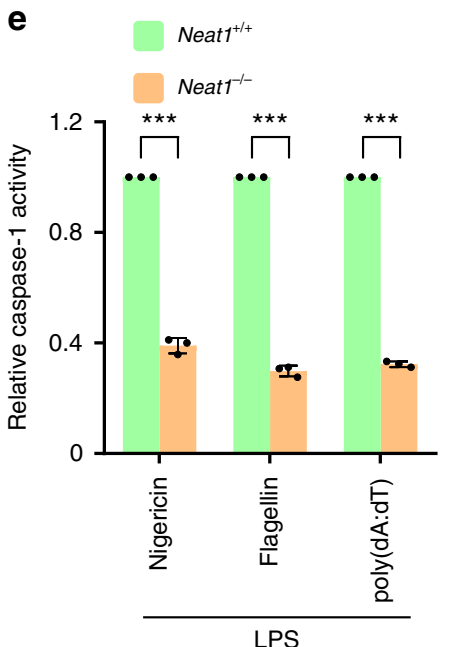

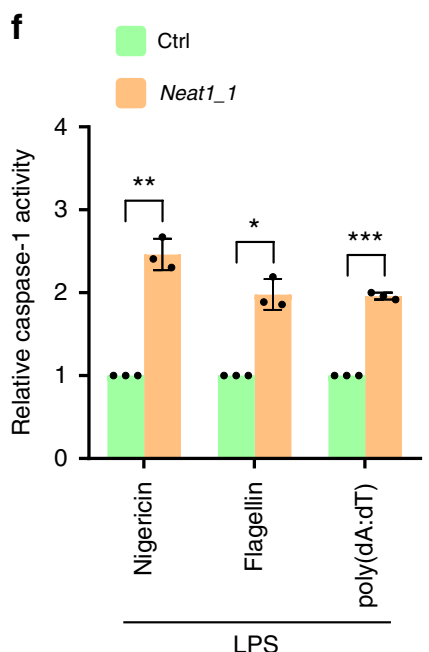

Fig. 5 Neat1 stabilizes the caspase-1 hetero-tetramers and increases caspase-1 activity. a-d 293T cells were infected with lentiviruses expressing control RNA or Neat1_1 and then co-transfected with Flag-p20 and HA-p10 (a, b) or Flag-p33 and HA-p10 (c, d). Cell lysates were incubated with anti-Flag mAb (M2) beads. Input samples and pull-down products were subjected to western blotting (a, c). Relative ratios of HA-p10 pulled down by Flag-p20 (b) or Flag-p33 (d) were shown in histogram, based on the data of three independent replication experiments. e Neat $7^{+/+}$and Neat $1^{-/-}$BMDMs were primed with LPS and then activated with nigericin, flagellin, or poly(dA:dT). Mature caspase-1 activity was measured. $\mathbf{f}$ Control or Neat1_1 overexpressing iBMDMs were primed with LPS and then activated with nigericin, flagellin, or poly(dA:dT). Mature caspase- 1 activity were measured. In $\mathbf{b}, \mathbf{d}, \mathbf{e}$ and $\mathbf{f}$, data shown are mean \pm SD $(n=3) .{ }^{\star} P<0.05,{ }^{\star \star} P<0.01,{ }^{\star \star \star} P<0.001$, two-tailed $t$-test. Source data are provided as a Source Data file 
a

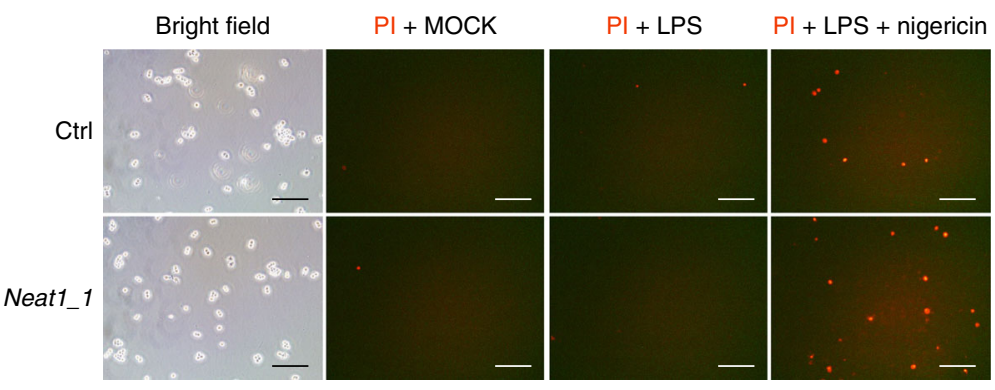

C

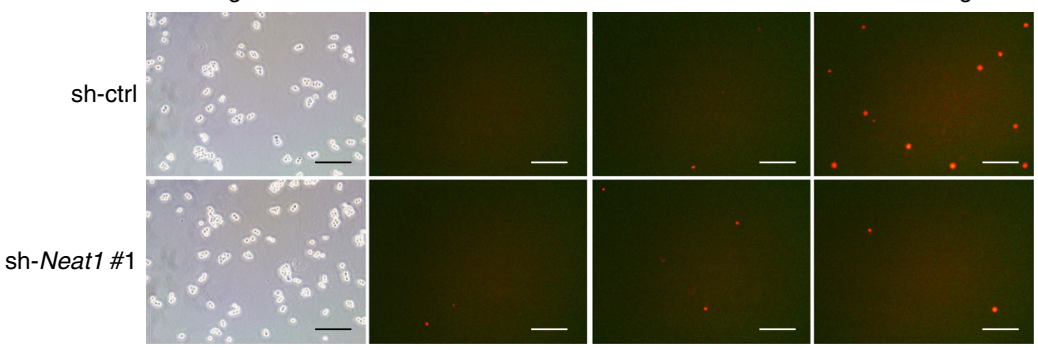

e

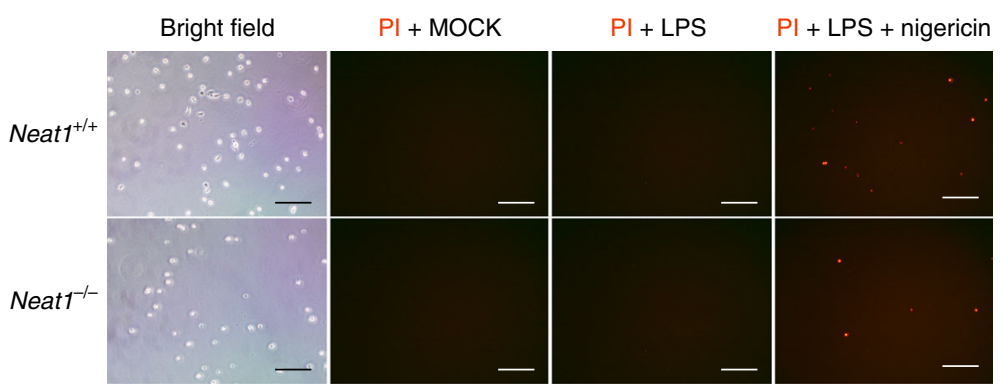

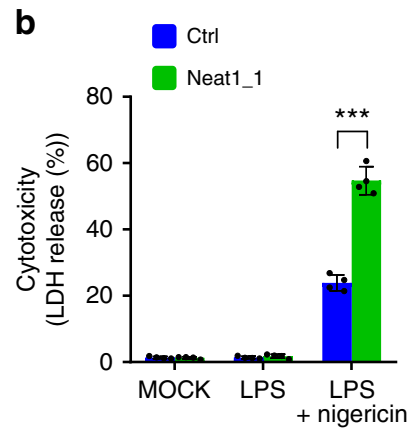

d
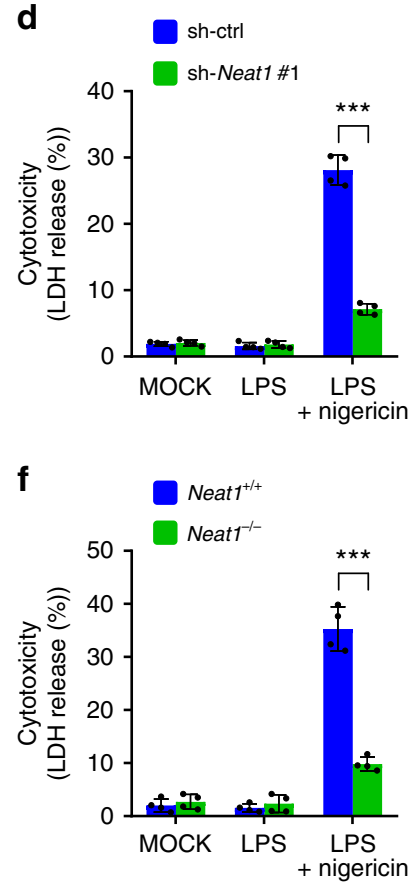

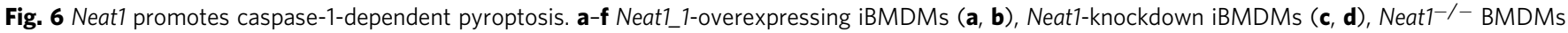
$(\mathbf{e}, \mathbf{f})$, and the corresponding control cells were primed with LPS and then stimulated with nigericin. Cells were stained with PI and analyzed under microscope (a, c, and $\mathbf{e}$ ). Percentages of pyroptotic cells were measured by LDH release into supernatants (b, d, and $\mathbf{f}$ ). In $\mathbf{a}, \mathbf{c}$, and $\mathbf{e}$, the bar graph indicates $300 \mu \mathrm{m}$. In $\mathbf{b}, \mathbf{d}$, and $\mathbf{f}$, data shown are mean \pm SD $(n=4)$. ${ }^{\star \star \star} P<0.001$, two-tailed $t$-test. Source data are provided as a Source Data file

isoform remained unchanged (Supplementary Fig. 7a). To understand how Neat 1 participates in the inflammasome activation, we examined the presence of Neat1 in the cytoplasmic fraction and culture medium of cells treated under different conditions. Compared with untreated or LPS-primed iBMDMs, LPS and nigericin co-stimulated iBMDMs contained more Neat1 in the cytoplasm $(\sim 8.5 \%$ versus $\sim 1 \%$; Fig. 7 a and Supplementary Fig. 7b). Moreover, consistent with the increased pyroptosis, LPS and nigericin co-stimulated iBMDMs also released more Neat 1 into the culture medium (Supplementary Fig. 7c). Similarly, more Neat 1 was released from nucleus to cytoplasm in LPS/flagellin- or LPS/poly(dA:dT)-treated iBMDMs compared to untreated or LPS-primed iBMDMs (Fig. 7b, c and Supplementary Fig. 7d, e). Additionally, we treated iBMDMs with LPS and 2DG to stimulate the formation of Nlrp1b inflammasome. This also led to the translocation of Neat 1 to the cytoplasm (Supplementary Fig. 7f, g). These observations suggest that Neat1 is released from nucleus to the cytoplasm during the activation of the NLRP3, NLRC4, AIM2, or Nlrp1b inflammasome.

To corroborate the observations, we performed RNA fluorescence in situ hybridization (RNA-FISH) to assess the cellular localization of Neat1. Among untreated or LPS-primed iBMDMs, Neat1 remained in the nucleus while ASC was diffusely localized in the cytoplasm (Fig. 7d). However, in LPS and nigericin costimulated iBMDMs where ASC became polymerized, a portion of Neat 1 was released to the cytoplasm and became colocalized with ASC (Fig. 7d), further supporting the notion that the nuclear export of Neat1 is induced in activated cells to facilitate inflammasomes formation.

Perturbation of paraspeckles leads to the release of Neat1. Neat 1 is concentrated in paraspeckles and is an essential architectural component of these nuclear bodies ${ }^{35,36}$. To examine how LPS and nigericin co-stimulation may induce Neat1 nuclear export, we examined levels of three main paraspeckle proteins, Nono, Pspc1, and Sfpq ${ }^{35}$. Compared with control or LPS-treated iBMDMs, Pspc1 and the short isoform of Sfpq (Sfpq-S) declined in their abundance in LPS and nigericin co-treated iBMDMs, while the levels of Nono and the long isoform of Sfpq (Sfpq-L) remained unchanged (Fig. 8a). Levels of Pspc1 and Sfpq-S were also decreased in iBMDMs activated by flagellin or poly(dA:dT) (Fig. 8b, c). In addition, levels of Pspc1 declined in 2DG-activated iBMDMs (Supplementary Fig. 8a). Accordingly, the size and number of paraspeckles were dramatically reduced in LPS and nigericin co-stimulated iBMDMS compared to untreated or 

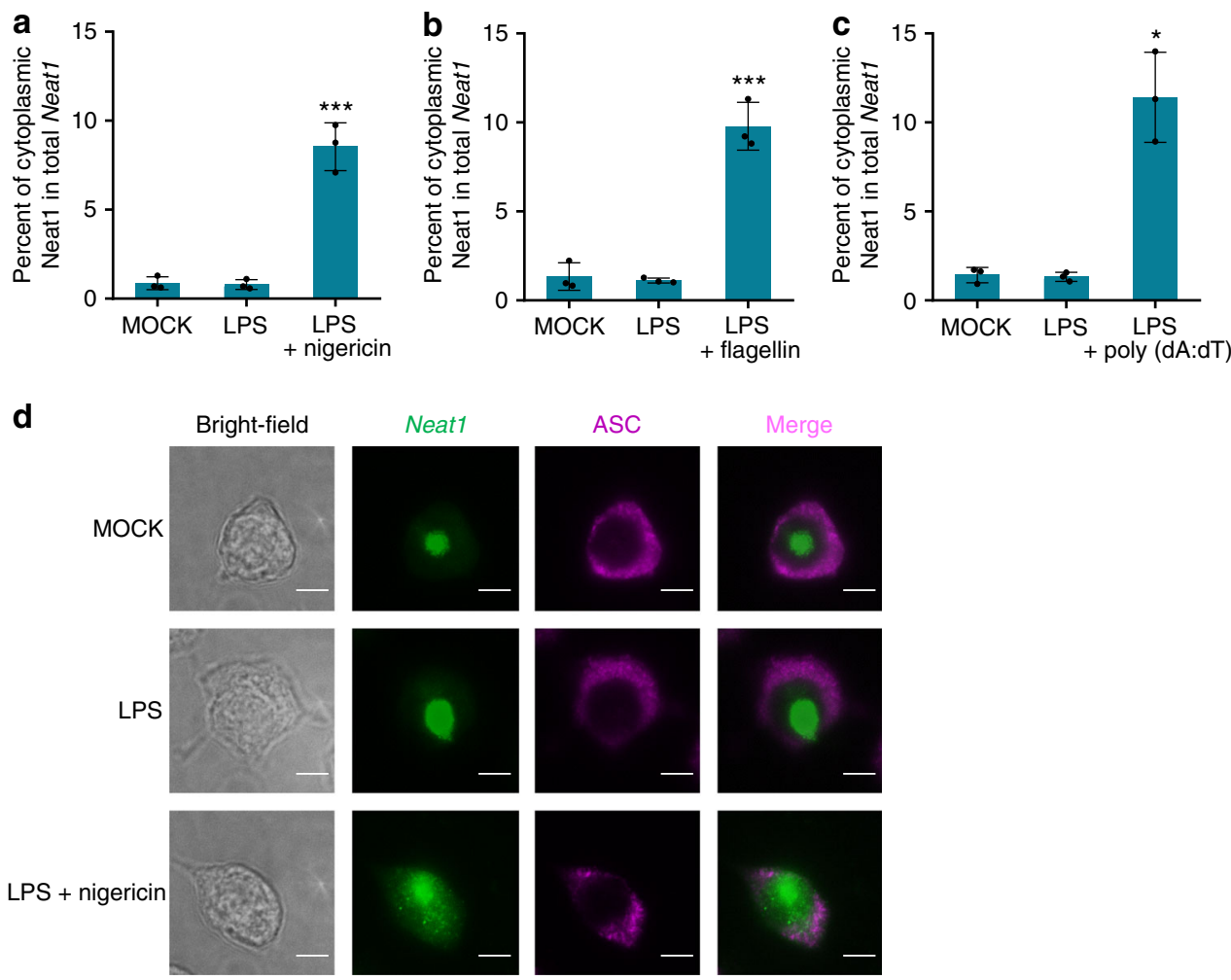

Fig. 7 Neat1 is exported from nucleus to cytoplasm in response to inflammasome-activating stimuli. a-c Untreated iBMDMs (a-c), LPS-primed iBMDMs (a-c), nigericin-activated iBMDMs (a), flagellin-activated iBMDMs (b), or poly(dA:dT)-activated iBMDMs (c) were subjected to fractionation into cytoplasmic and nuclear extracts. Cytoplasmic RNA was analyzed for the Neat1 expression by real-time RT-PCR. $\mathbf{d}$ The subcellular co-localization of Neat1 (green) and ASC (purple) in untreated, LPS-primed or LPS-nigericin-co-stimulated iBMDMs was analyzed by RNA-FISH and IF. In d, the bar graph indicates $5 \mu \mathrm{m}$. In $\mathbf{a}, \mathbf{b}$, and $\mathbf{c}$, data shown are mean \pm SD $(n=3)$. ${ }^{\star} P<0.05$, ${ }^{\star \star \star} P<0.001$, two-tailed $t$-test. Source data are provided as a Source Data file

LPS-primed iBMDMs (Fig. 8d and Supplementary Fig. 8b, c). Consistently, the interaction of Nono with Pspcl and Sfpq was reduced in response to the activation of the NLRC4, AIM2, and Nlrp1b inflammasome (Fig. 8e, f and Supplementary Fig. 8d). All above results claim that activation of various inflammasomes weakens assembly of paraspeckles, which store Neat1 in nucleus.

To verify that the perturbation of paraspeckles results in the release of Neat1 into the cytoplasm, we knocked down Nono, Pspc1, and Sfpq individually (Supplementary Fig. 8e, f). In each case, the nuclear export of both total Neat1 and Neat_2 was observed, and a substantial fraction of Neat1 ( 15-20\%) was found in the cytoplasm (Fig. 8g, h). Therefore, stimuli, that activate various inflammasomes, disrupt paraspeckles leading to the translocation of Neat 1 to the cytoplasm, where Neat1 influences the assembly of inflammasomes.

Neat1 mediates the effect of HIF-2a on inflammasomes. The expression of Neat1 is induced by infection of viruses (e.g., influenza virus and herpes simplex virus $)^{49,50}$, and is also regulated by the tumor suppressor $\mathrm{p} 53^{51}$, a central sentinel for various internal and external stresses ${ }^{52,53}$. In addition, hypoxia, which promotes inflammation via HIF-1 $\alpha$-mediated induction of NLRP3 and IL-1 $\beta^{24,25}$, can induce Neat1 through HIF-2 $\alpha$ mediated transcriptional activation ${ }^{54,55}$. Thus, Neat 1 may function as a stress sensor for associated inflammasomes. To test this possibility, we investigated the role of Neat 1 in hypoxia-mediated activation of the NLRP3 inflammasome. iBMDMs cultured under hypoxic conditions exhibited increased levels of Neat1 (Supplementary Fig. 9a), HIF-1 $\alpha$, HIF-2 $\alpha$, p65, NLRP3, and IL-1 $\beta$ (Fig. 9a), which was accompanied by enhanced NLRP3 inflammasome formation, caspase- 1 activation, and IL- $1 \beta$ secretion
(Fig. 9a). However, knockout of Neat1 not only reduced the activation of NLRP3 inflammasome under normoxic conditions, but also effectively prevented the increase of inflammasome activation under hypoxic conditions (Supplementary Fig. 9b), underscoring a critical role for Neat1 in hypoxia-induced NLRP3 inflammasome activation. In addition, activation of NLRC4 and AIM2 inflammasomes was also increased under hypoxic conditions, along with increased expression of HIF-1 $\alpha$, HIF-2 $\alpha$, and IL$1 \beta$ (Fig. 9b, c). Knocking down either HIF-1a (Fig. 9d, f, h) or HIF-2 $\alpha$ (Fig. 9e, g, i) reduced hypoxia-induced caspase-1 activation and IL-1 $\beta$ maturation under the condition of NLRP3, NLRC4, or AIM2 inflammasome activation (Fig. 9d-i), indicating that both HIF-1 $\alpha$ and HIF-2 $\alpha$ mediate the effect of hypoxia on canonical inflammasomes. HIF-1 $\alpha$ knockdown, but not HIF-2 $\alpha$ knockdown, reduced the activation of diverse inflammasomes in iBMDMs devoid of Neat1 (Fig. 9d-i), which claims that Neat1silencing could efficiently block the effect of HIF-2 $\alpha$, but not HIF$1 \alpha$, on inflammasomes activation. These results suggest that hypoxia-induced activation of the NLRP3, NLRC4, and AIM2 inflammasomes and associated inflammatory responses are in part due to HIF-2a-mediated upregulation of Neat1.

Loss of Neat1 reduces inflammation in vivo. To investigate the effect of Neat1 in vivo, we used an NLRP3 inflammasome-mediated, IL-1-dependent mouse peritonitis model induced by the aluminum salt alum ${ }^{56}$. We intraperitoneal (i.p.) injected alum in Neat $1^{+/+}$and $N e a t 1^{-/-}$mice to induce peritonitis, and assayed IL- $1 \beta$ in the lavage fluid. A significant reduction of IL- $1 \beta$ secretion in the lavage fluid was observed in Neat $1^{-1-}$ mice compared to Neat $1^{+/+}$mice (by $\sim 67 \%$; Fig. 10a). Moreover, collected peritoneal exudate cells (PECs) and analyzed alum-induced recruitment of 
a

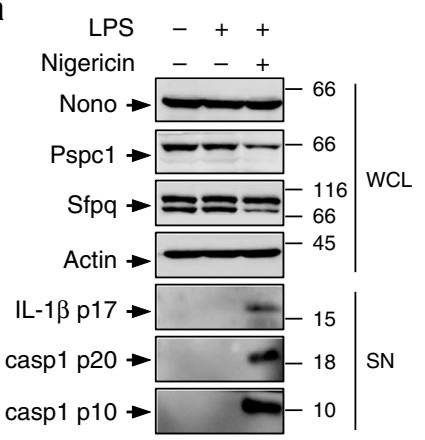

b

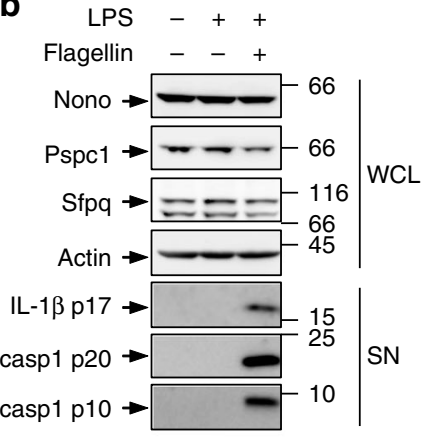

d
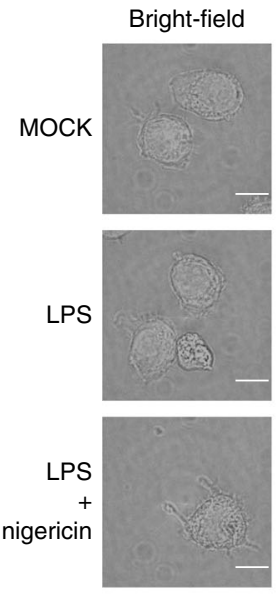

Sfpq
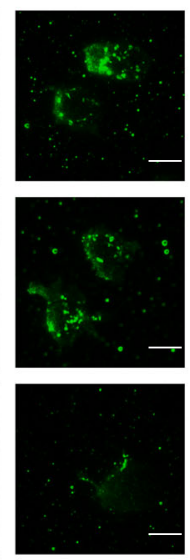

Pspc1
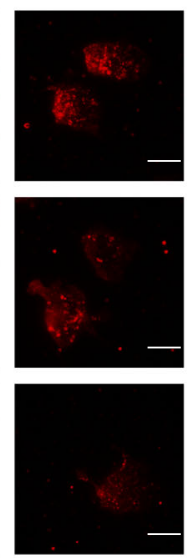
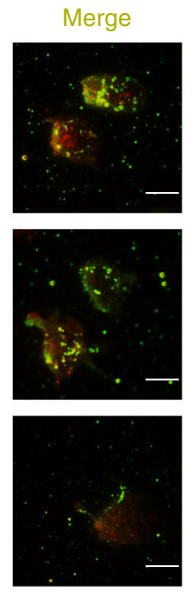

g

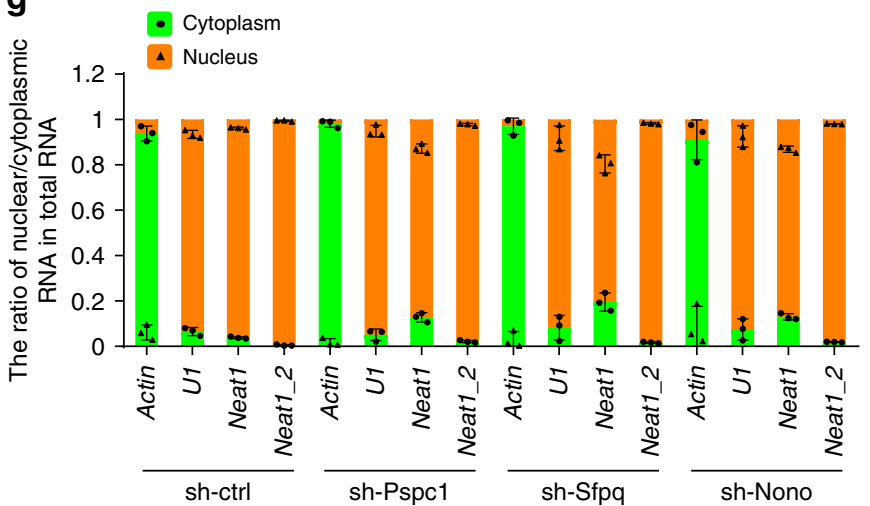

c

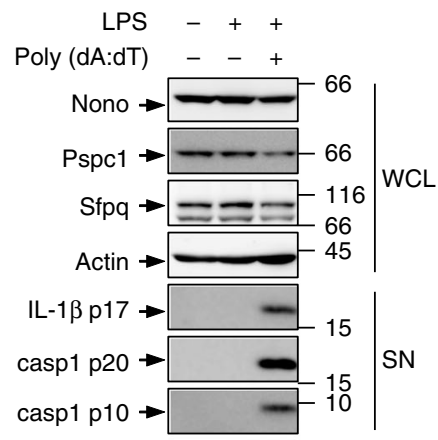

e

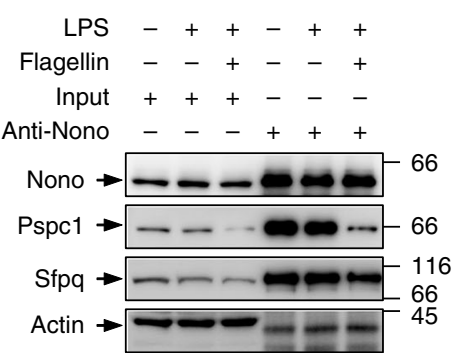

f LPS -++-++

Poly (dA:dT) - -+--+

Input +++--

Anti-Nono $-c_{-}+++$

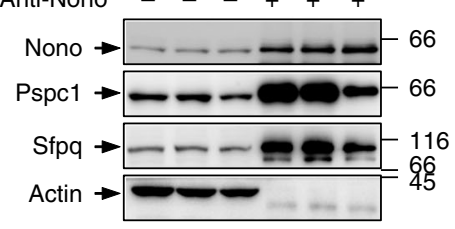

h

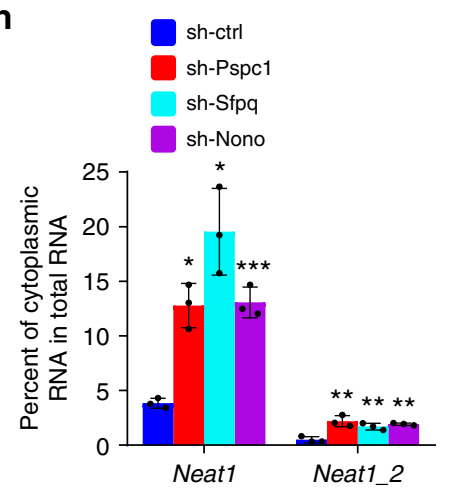

Fig. 8 Neat1 is released from nuclear paraspeckles into cytoplasm during inflammasome activation. a-c Cell lysates of untreated iBMDMs (a-c), LPSprimed iBMDMs (a-c), nigericin-activated iBMDMs (a), flagellin-activated iBMDMs (b), or poly (dA:dT)-activated iBMDMs (c) were analyzed by Western blotting. d Colocation of paraspeckle proteins, Sfpq (green), and Pspc1 (red) were assayed by immunostaining. The location of paraspeckles (yellow) was obtained by merging two signals of Sfpq and Pspc1. The position of the nucleus is shown in the bright-field image. e, $\mathbf{f}$ Interactions among paraspeckle components in untreated iBMDMs $(\mathbf{e}, \mathbf{f})$, LPS-primed iBMDMs $(\mathbf{e}, \mathbf{f})$, flagellin-activated iBMDMs $(\mathbf{e})$, or poly (dA:dT)-activated iBMDMs $(\mathbf{f})$ were analyzed by co-immunoprecipitation assay. $\mathbf{g}, \mathbf{h}$ iBMDMs infected with lentiviruses expressing control, Pspc1, Sfpq, or Nono shRNA were analyzed for the cytoplasmic and nuclear levels of Neat1 and Neat1_2. Percentages of cytoplasmic Neat1 or Neat1_2 among total Neat1 or Neat1_2 RNA were shown in h. In d, the bar graph indicates $10 \mu \mathrm{m}$. In $\mathbf{g}$ and $\mathbf{h}$, data shown are mean $\pm \operatorname{SD}(n=3) .{ }^{\star} P<0.05,{ }^{\star \star} P<0.01,{ }^{\star \star \star} P<0.001$, two-tailed $t$-test. Source data are provided as a Source Data file

inflammatory cells by flow cytometry (Supplementary Fig. 10a). The number of total PECs recruited upon alum challenge was markedly decreased by $58 \%$ in Neat $1^{-1-}$ mice (Fig. 10b). Aluminduced recruitment of neutrophils and $\mathrm{Ly} 6 \mathrm{C}^{+}$monocytes was also strongly inhibited in the Neat $1^{-1-}$ mice (Fig. 10c, d).
To evaluate the role of Neat1 on NLRC4 inflammasome activation in vivo, we used a mouse model of flagellin-induced pneumonia. Neat $1^{+/+}$and Neat1 $1^{-/-}$mice were intranasally instilled with TurboFect reagent and flagellin to induce pneumonia. We found that IL-1 $\beta$ secretion in bronchoalveolar 
a

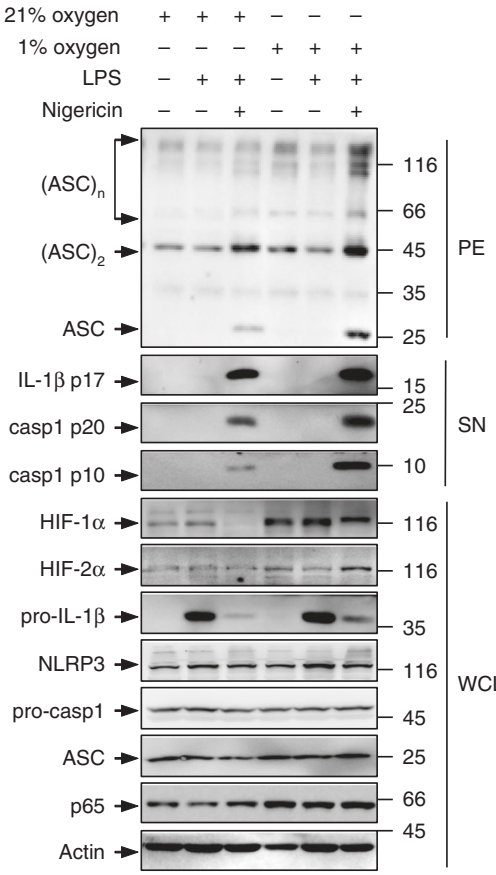

$\mathbf{f}_{\text {sh-Neat1\#1 }}-\quad+\quad++$

sh-HIF-1 $\alpha \quad++$

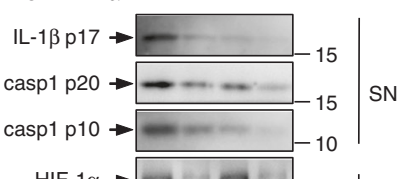

$\mathrm{HIF-1} \alpha \rightarrow-116$

$\mathrm{p} 65 \rightarrow-2-66$

$\mathrm{NLRC4} \rightarrow-1-\ldots$

ASC $\rightarrow---25$ WCL

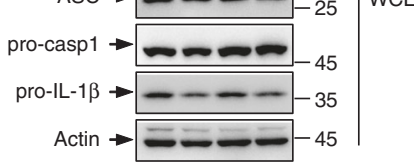

Hypoxia + LPS + flagellin

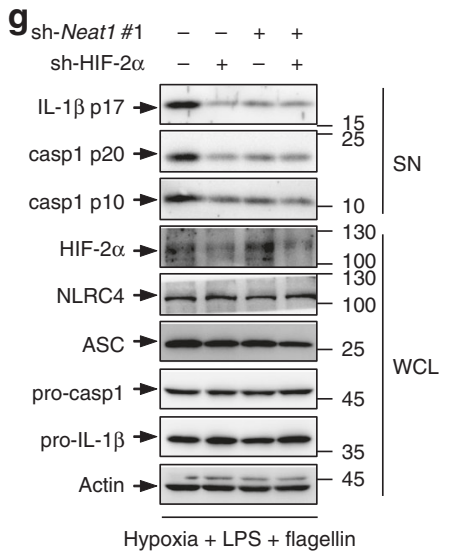

b

$21 \%$ oxygen +++--

$1 \%$ oxygen --+++

LPS -++-+

Flagellin $-\ldots+--+$

$\mathrm{IL}-1 \beta \mathrm{p} 17 \rightarrow$
casp1 $\mathrm{p} 20 \rightarrow-15$
-10

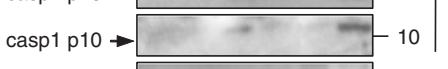

$\mathrm{HIF-} 1 \alpha \rightarrow-116$
$\mathrm{HIF}-2 \alpha \rightarrow-116$

$\begin{aligned} \text { HIF-2 } \alpha \rightarrow & \\ \text { pro-IL-1 } \beta \rightarrow \longrightarrow-\infty & \end{aligned}$

NLRC4 $\rightarrow-1-116$ WCL

pro-casp1 $\rightarrow-(45$

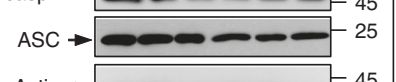

Actin $\rightarrow-\longrightarrow-45$

d

$\begin{array}{rrr}\text { Sh-Neat1\#1 } & - & + \\ \text { sh-HIF-1 } \alpha & - & +\end{array}$

$\begin{aligned} \mathrm{IL}-1 \beta \mathrm{p} 17 & \rightarrow- \\ \text { casp1 p20 } & \rightarrow-15 \\ \text { casp1 p10 } \rightarrow-\text { SN } & \rightarrow-10\end{aligned}$

HIF- $1 \alpha \rightarrow=-116$

p65 $\rightarrow--66$

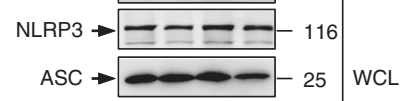

pro-casp1 $\rightarrow-\ldots-45$

pro-IL-1 $\rightarrow-\square-35$

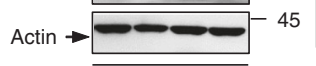

Hypoxia + LPS + nigericin

h

sh-Neat1\#1 - - + +

sh-HIF-1 $1 \alpha \quad+\quad+$

$\begin{aligned} \mathrm{IL}-1 \beta \mathrm{p} 17 & \rightarrow-15 \\ \text { casp1 } \mathrm{p} 20 \rightarrow-\infty-25 & \rightarrow \mathrm{SN}\end{aligned}$

casp1 p10 $\rightarrow-10$

$\mathrm{HIF-1} \alpha \rightarrow=116$

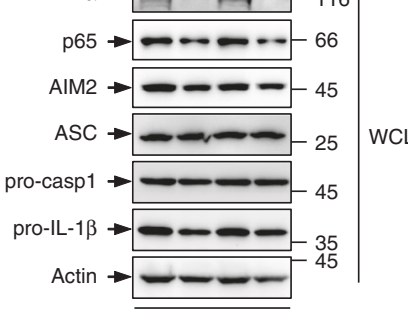

Hypoxia + LPS + poly (dA:dT)
C

$21 \%$ oxygen +++--

$1 \%$ oxygen --++

Poly (dA:dT) - $\quad+\ldots+$

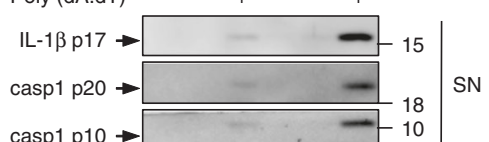

casp1 p10 $\rightarrow \longrightarrow-18$

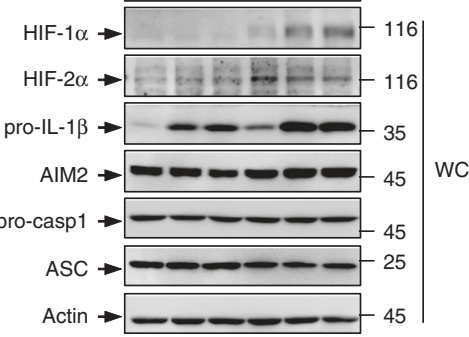

e

Sh-Neat1 \#1 $-\quad++$

sh-HIF-2 $\alpha-++$

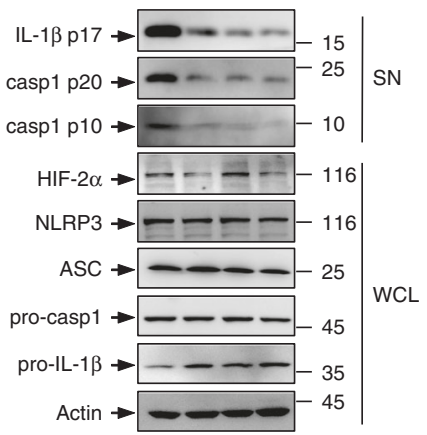

Hypoxia + LPS + nigericin

i

Ih-Neat1\#1 - -++

sh-HIF-2 $\alpha-++$

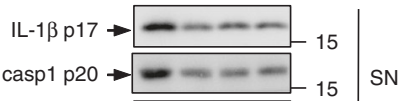

casp1 p10 $\rightarrow--15$

HIF-2 $\alpha \rightarrow+11+-116$

AIM2 $\rightarrow--45$

ASC $\rightarrow=-25$

pro-casp1 $\rightarrow-(-25$ WCL

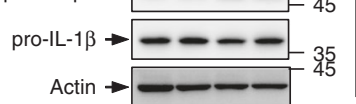

Hypoxia + LPS + poly (dA:dT)

\section{N}

L 
a
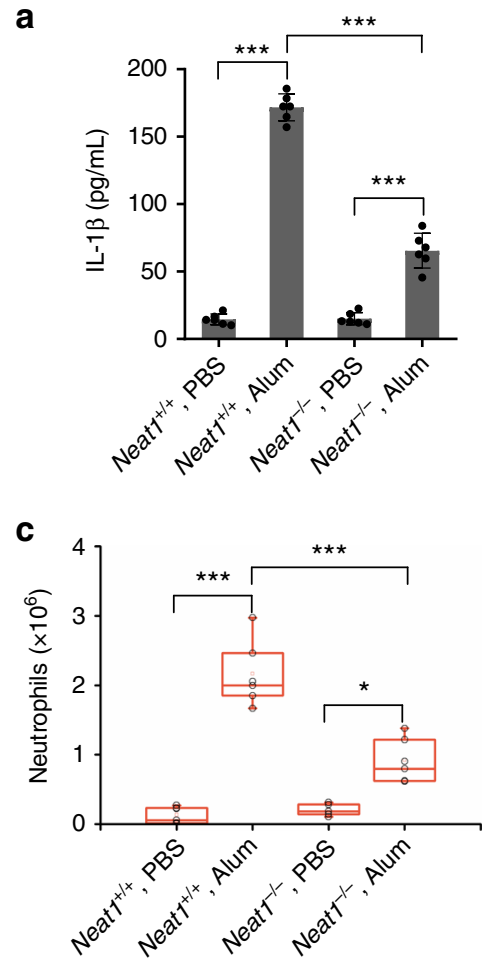

e
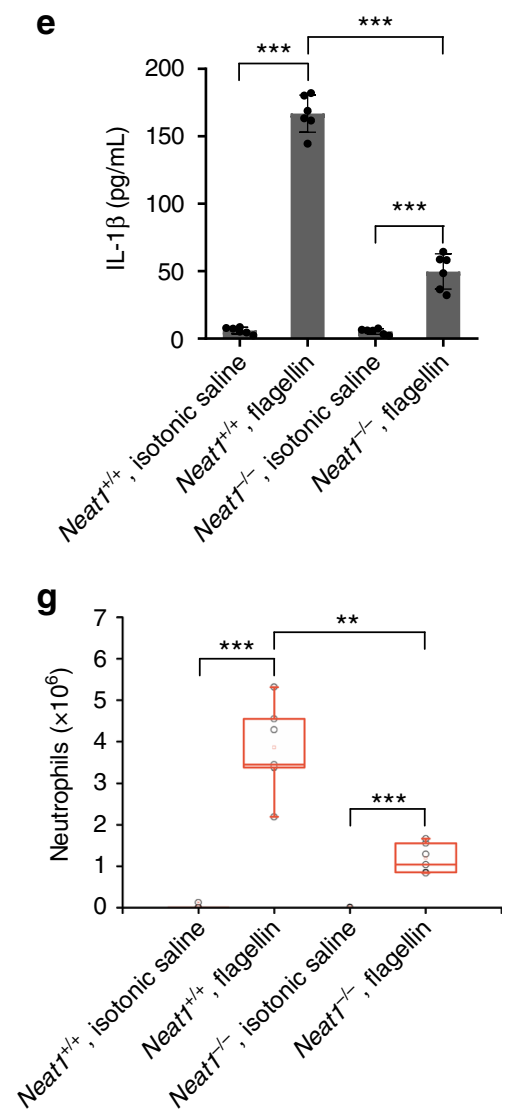

b

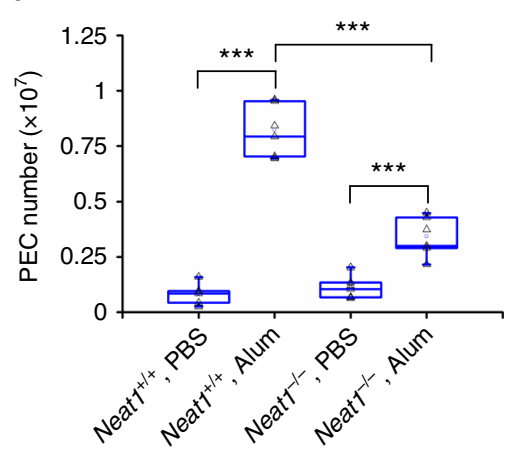

d

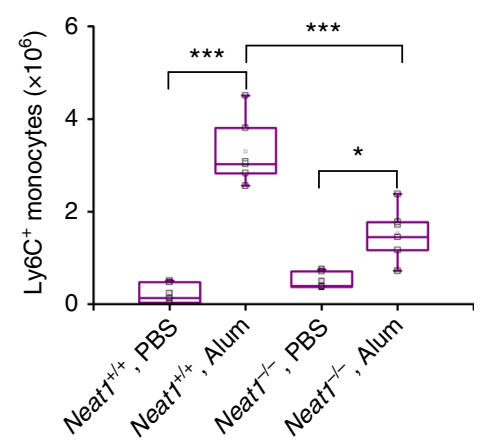

f

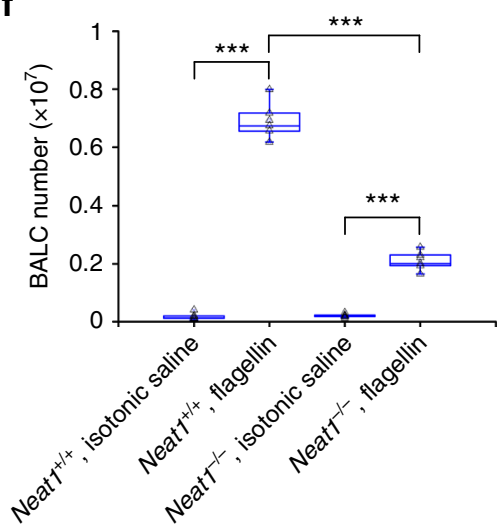

h

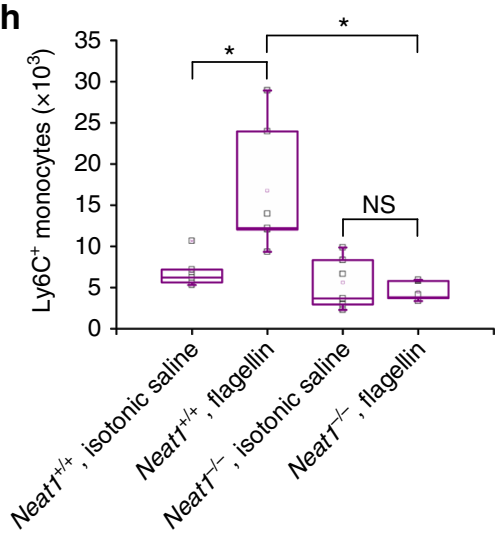

Fig. 10 Loss of Neat 1 impairs alum-induced peritoneal inflammation and attenuates flagellin-induced lung inflammation in vivo. a IL-1 13 content in the lavage fluid from Neat $1^{+/+}$or Neat $1^{-/-}$mice $12 \mathrm{~h}$ after PBS or alum injection. b Absolute numbers of Neat $1^{+/+}$or Neat $1^{-/-}$PECs recovered $12 \mathrm{~h}$ after PBS or alum injection. c, d Absolute numbers of neutrophils (c) and Ly6C+ monocytes (d) exuded to the peritoneum $12 \mathrm{~h}$ after PBS or alum injection. $\mathbf{e}$ IL-1 $\beta$ content in the BALF from Neat ${ }^{1 / /+}$ or Neat $7^{-/-}$mice $12 \mathrm{~h}$ after isotonic saline or flagellin intranasal instillation. $\mathbf{f}$ Absolute numbers of Neat ${ }^{+/+}$or Neat $7^{-/-}$PECs recovered $12 \mathrm{~h}$ after isotonic saline or flagellin intranasal instillation. $\mathbf{g}$, $\mathbf{h}$ Absolute numbers of neutrophils $(\mathbf{g})$ and Ly $6 \mathrm{C}^{+}$monocytes $(\mathbf{h})$ exuded to the lung $12 \mathrm{~h}$ after isotonic saline or flagellin intranasal instillation. In a-h, data shown are mean $\pm \mathrm{SD}(n=6) .{ }^{\star} P<0.05,{ }^{\star \star} P<0.01,{ }^{\star \star \star} P<0.001$, two-tailed $t$-test. Source data are provided as a Source Data file 
lavage fluid (BALF) was decreased by $70 \%$ in flagellin-challenged Neat $1^{-1-}$ mice compared to flagellin-challenged $\mathrm{Neat}^{+/+}$mice (Fig. 10e). We also analyzed bronchoalveolar lavage cells (BALCs) and flagellin-induced recruitment of inflammatory cells by flow cytometry (Supplementary Fig. 10b). The total number of BALCs recruited upon flagellin challenge was decreased by $70 \%$ in Neat $1^{-1-}$ mice (Fig. 10f). Furthermore, flagellin-induced recruitment of neutrophils and $\mathrm{Ly}_{6} \mathrm{C}^{+}$monocytes was strongly decreased in Neat $1^{-1-}$ mice (Fig. 10g, h).

The overt defects of Neat $1^{-1-}$ mice in alum-induced peritoneal inflammation and flagellin-induced lung inflammation emphasize an important function for Neat1 in the activations of the canonical inflammasomes, the subsequent accumulation of immune cells and the associated symptoms in vivo.

\section{Discussion}

Proper control of inflammasome assembly and caspase- 1 activation permits effective antimicrobial and inflammatory responses while avoiding tissue damage. Yet, how these supramolecular assemblies are regulation remains incompletely defined. LncRNAs are increasingly recognized as important regulators in different biological settings, but few lncRNAs are implicated in innate immunity $57-60$. The current work reveals that the lncRNA Neat 1 plays a critical role in regulating various caspase-1-associated canonical inflammasomes including NLRC4, AIM2, and especially NLRP3 inflammasomes. Neat1 promotes the assembly of inflammasomes, and also stabilizes the mature caspase- 1 tetramers, $(\mathrm{p} 20: \mathrm{p} 10)_{2}$, and (p33:p10) ${ }_{2}$, and increases their protease activity. Thus, Neat 1 is likely a previously unanticipated RNA component that enables the assembly of these largely proteinaceous signaling platforms to facilitate the recruitment, maturation, and stabilization of caspase-1 in activated macrophages. The ability of Neat1 to directly regulate inflammasomes provides a notable example for the role of lncRNAs in regulating large multimeric protein complexes in the cytoplasm.

The assembly of inflammasomes requires a common priming event, such as LPS-induced activation of TLRs, which induces the expression of NLRP3 and pro-IL-1 $\beta^{2,5}$. However, the NLRC4, AIM2, and especially NLRP3 inflammasomes are versatile inflammasome, collectively responding to a wide range of stimuli ${ }^{5,24,61-64}$. It remains unclear whether these stimuli also employ a general mechanism to modulate inflammasomes. Of note, the expression of Neat1 is highly regulated by various signals, including those that activates the tumor suppressor p53 (refs. ${ }^{40,51}$ ). Moreover, hypoxia is a key physiological stressor present within both normal and tumor microenvironments ${ }^{65}$ and a critical determinant of the immune response. Here we show that Neat 1 links the effect of hypoxia with the activation of NLRP3, NLRC4, and AIM2 inflammasomes. Both human NEAT1 and murine Neat 1 are thought to maintain the structural integrity of the paraspeckles ${ }^{35}$, the function of which remains unclear. We find that the paraspeckles are disrupted in response to various inflammasome-activating signals, leading to the release of Neat 1 and subsequent translocation to the cytoplasm, implying that paraspeckles may serve as a storage depot for Neat1. Therefore, the upregulation and translocation of Neat1 may be a converging event leading to the activation of the various caspase-1-associated canonical inflammasomes by a wide range of stimuli, underscoring a common mechanism for the assembly of these inflammation signaling platforms. In addition, our finding, along with previous observation that ASC and NLRP3 are recruited from other cellular components to mitochondria and associated endoplasmic reticulum membranes upon both priming and activation processes ${ }^{66}$, also highlights the trafficking of inflammasome components as a major mechanism for the regulation of inflammasome activity.

Finally, by generating Neat 1 knockout mice and analyzing their response to alum-induced peritoneal inflammation and flagellininduced lung inflammation, we demonstrate that Neat1 enhances the activation of NLRP3 and NLRC4 inflammasomes and promotes pyroptosis in vivo (Supplementary Fig. 11). Therefore, Neat1 may represent a therapeutic target for inflammasomeassociated diseases such as gout and autoinflammatory syndromes.

\section{Methods}

Antibodies and reagents. Antibodies used for western bloting included anti-IL-1 $\beta$ (R\&D Systems, 1:1000, AF-401-NA), anti-HIF-2 $\alpha$ (R\&D Systems, 1:1000, AF2997) anti-NLRP3 (Adipogen, 1:1000, AG-20B-0014), anti-ASC (Adipogen, 1:1000, AG25B-0006), anti-caspase-1 (p20) (Adipogen, 1:1000, AG-20B-0042), anti-caspase-1 (p10) (Adipogen, 1:1000, AG-20B-0044), anti-p65 (Cell Signaling Technology, 1:1000, 8242), anti-HIF-1a (Cell Signaling Technology, 1:1000, 14179), anti-AIM2 (Cell Signaling Technology, 1:1000, 13095), anti-IL-6 (Cell Signaling Technology, 1:1000, 12912), anti-Actin (CMC-TAG, 1:2000, AT0009), anti-FLAG (SigmaAldrich, 1:2000, F3165), anti-Pspc1 (Santa Cruz, 1:200, sc-374181), anti-Sfpq (Santa Cruz, 1:200, sc-271796), anti-Nono (ABclonal, 1:500, A5282), anti-NLRC4 (ABclonal, 1:500, A7382), anti-histone H2A (abcam, 1:1000, ab177308), HRPlinked anti-rabbit IgG (Cell Signaling Technology, 1:2000, 7074), HRP-linked antimouse IgG (Cell Signaling Technology, 1:2000, 7076), HRP-linked anti-goat IgG (Santa Cruz, 1:1000, sc-2354). Antibodies used for immunoprecipitation included anti-NLRP3 (Cell Signaling Technology, 1:200, 15101), anti-caspase-1 (p20) (Adipogen, 1:200, AG-20B-0042), anti-ASC (Adipogen, 1:200, AG-25B-0006), antiNono (ABclonal, 1:50, A5282), normal rabbit IgG (Cell Signaling Technology, 1:200, 2729), normal mouse IgG (Santa Cruz, 1:80, sc-2025). Anti-ASC (Santa Cruz, 1:50, sc-22514), anti-Pspc1 (mouse IgG, Santa Cruz, 1:50, sc-374181), antiSfpq (mouse IgG, Santa Cruz, 1:50, sc-271796), anti-Sfpq (rabbit IgG, Proteintech, 1:50, 15585-1-AP), anti-Nono (rabbit IgG, ABclonal, 1:50, A5282), Alexa fluor 488 donkey anti-rabbit IgG(H $+\mathrm{L})$ (Life Technologies, 1:1000, A21206), Alexa fluor 568 donkey anti-mouse $\operatorname{IgG}(\mathrm{H}+\mathrm{L})$ (Life Technologies, 1:1000, A10037), and Alexa fluor 647 donkey anti-rabbit IgG(H + L) (Life Technologies, 1:1000, A31573) were used for immunofluorescence. PerCP-Cy ${ }_{5.5}$ anti-mouse CD11b (BD Biosciences, 1:1000, 550993), APC anti-mouse Ly-6G (BD Biosciences, 1:1000, 560599), and PE anti-mouse Ly-6C (BD Biosciences, 1:1000, 560592) were used for flow cytometry. LPS, nigericin, flagellin, and poly(dA:dT) were purchased from Invivogen; Lipofectamine 2000 Transfection Reagent was from Invitrogen; DOTAP Liposomal Transfection Reagent was from Roche; puromycin, MG132, propidium iodide, and 2-deoxy-D-Glucose were from Solarbio; proteinase $\mathrm{K}$ and RiboLock RNase Inhibitor were from ThermoFisher Scientific; and Igepal CA-630 was from Sigma. Caspase-1 Activity Assay Kit purchased from Beoytime was used to perform the semi-quantitative analysis of mature caspase-1 activity.

Mice. Neat $1^{-I-}$ mice on a C57BL/6 background were generated by Biocytogen Biological Technology Co., Ltd. Briefly, C57BL/6 mice and Kunming (KM) mice were housed in a specific pathogen free facility. For Neat1 targeting, two sgRNAs (Supplementary Table 1) were designed to target either a region upstream or downstream of the gene Neat1. For each targeting site, candidate guide RNAs were designed by the CRISPR design tool (http://crispr.mit.edu). Guide RNAs were screened for on-target activity use UCATM. UCATM (Universal CRISPR Activity Assay), a sgRNA activity detection system developed by Biocytogen, is simpler and more sensitive than MSDase assay. The Cas9 mRNA and sgRNAs were transcribed by T7 RNA polymerase in vitro. For Cas 9 mRNA and sgRNAs production, add the T7 promoter sequence to the Cas9 or sgRNA template by PCR amplification. The T7-Cas/sgRNA PCR products was gel purified and used as the template for in vitro transcription using the MEGA shortscript T7 kit (Life Technologies) according to the kit protocol. Purify the Cas 9 mRNA and sgRNAs using the MEGAclear kit and elute with RNase-free water. C57BL/6 female mice and $\mathrm{KM}$ mouse strains were used as embryo donors and pseudopregnant foster mothers, respectively. Superovulated female C57BL/6 mice (3-4 weeks old) were mated to C57BL/6 stud males, and fertilized embryos were collected from the ampullae of super ovulated. Different concentrations of Cas 9 mRNA and sgRNAs were mixed and co-injected into the cytoplasm of one-cell stage fertilized eggs. After injection, surviving zygotes were transferred into oviducts of KM albino pseudopregnant females. Sixty-five pups were born and then identified by genotyping with tail DNA. Neat $1^{+/-}$mice were used furtherly to reproduce the next generation, which includes Neat $1^{-1-}$ mice and Neat $1^{+1+}$ mice. IL-6 $6^{-I-}$ mice were provided by professor Zexiong Lian's laboratory. Genotyping PCR primers sequences are shown in Supplementary Table 1. All animal experiments were undertaken and conducted with approval from the Animal Research Ethics Committee of the University of Science and Technology of China (Permit Number: USTCACUC1801057). 
Cell culture. IBMDM, L929, and 293T cell lines were cultured in DMEM (Dulbecco's modified Eagle's medium) medium containing 10\% fetal bovine serum. iBMDM and L929 cell lines were kindly provided by professor Rongbin Zhou. 293T cell lines (ATCC CRL-3216) were purchased from the American Type Culture Collection (ATCC, Manassas, VA, USA). All cells were tested for mycoplasma contamination by Cell Culture Contamination Detection Kit (ThermoFisher)

Cell stimulation. Macrophages were cultured in DMEM complemented with 10\% FBS, $1 \mathrm{mM}$ sodium pyruvate, and $2 \mathrm{mM} \mathrm{L}$-glutamine overnight, and the medium was changed to opti-MEM in the following morning. Cells were primed with LPS $(100 \mathrm{ng} / \mathrm{mL})$ for $3 \mathrm{~h}$, and subsequently stimulated with nigericin $(5 \mu \mathrm{M})$ for $0.5 \mathrm{~h}$ to activate NLRP3 inflammasome, DOTAP-transfected flagellin $(0.5 \mu \mathrm{g} / \mathrm{mL})$ for $3 \mathrm{~h}$ to activate NLRC4 inflammasome, Lipofectamine 2000-transfected poly(dA:dT) (1 $\mu \mathrm{g} / \mathrm{mL})$ for $3 \mathrm{~h}$ to activate AIM2 inflammasome or $2 \mathrm{DG}(50 \mathrm{mM})$ for $4 \mathrm{~h}$ to activate Nlrplb inflammasome. Total cell lysates, precipitated supernatants, and pellets of cells at different stages were analyzed by immunoblotting.

CLIP sequencing. CLIP was performed as previously described ${ }^{67,68}$. Briefly, two $10-\mathrm{cm}$ dishes of iBMDM cells were stimulated with LPS for $3 \mathrm{~h}$ followed by treated with nigericin for $0.5 \mathrm{~h}$. Activated iBMDMs were washed three times with cold PBS, and irradiated at $150 \mathrm{~mJ} / \mathrm{cm}^{2}$ at $254 \mathrm{~nm}$ in HL-2000 HybriLinker ${ }^{\mathrm{TM}} \mathrm{UV}$ Crosslinker. Cells were collected and resuspended in $1 \mathrm{ml}$ lysis buffer $(50 \mathrm{mM}$ Tris-HCl [pH 7.4], $100 \mathrm{mM} \mathrm{NaCl}, 1 \%$ Igepal CA-630, 0.1\% SDS, $0.5 \%$ sodium deoxycholate, $1 / 25$ volume of Roche Protease Inhibitor Cocktail, $40 \mathrm{U} / \mathrm{mL}$ RNase inhibitor, $20 \mu \mathrm{M}$ MG132) for 20 min Cell lysates were precleared with protein A/G beads (Pierce), and then were incubated with protein $A / G$ beads coated with NLRP3 antibody at $4{ }^{\circ} \mathrm{C}$ for $3 \mathrm{~h}$. After extensive washing with washing buffer (50 mM Tris-HCl [pH 7.4], $100 \mathrm{mM} \mathrm{NaCl}, 0.2 \%$ Igepal CA-630, 0.1\% SDS, $0.5 \%$ sodium deoxycholate, $40 \mathrm{U} / \mathrm{mL}$ RNase inhibitor), the bead-bound immunocomplexes were eluted using elution buffer $(50 \mathrm{mM}$ Tris- $\mathrm{HCl}, \mathrm{pH} 8.0,1 \%$ SDS, and $10 \mathrm{mM}$ EDTA) at $65^{\circ} \mathrm{C}$ for $10 \mathrm{~min}$. To isolate protein-associated RNAs from the eluted immunocomplexes, samples were treated with proteinase $\mathrm{K}$, and RNAs were extracted by phenol/chloroform. The RNA sequencing was performed and analyzed by KangChen Bio-tech, Shanghai, China. The sequencing data were deposited in the National Center for Biotechnology Information Gene Expression Omnibus database (GSE118722). Visualization analysis was performed by Integrative Genomics Viewer (IGV).

Propidium iodide staining and imaging. To examine pyroptotic cells, cells were treated as indicated in 12-well plates for image capture. Propidium iodide $(1 \mu \mathrm{g} /$ $\mathrm{mL}$ ) was added to the medium for monitoring cell membrane integrity. Static bright-field images of pyroptotic cells were captured using an Olympus IX71.

Immunoprecipitation and RNA pull-down. For immunoprecipitation assay, the lysates of iBMDMs were incubated with $2 \mu \mathrm{g} / \mathrm{ml}$ antibody recognizing particular protein or normal rabbit/mouse IgG at $4{ }^{\circ} \mathrm{C}$ for $2 \mathrm{~h}$, and then with $20 \mu \mathrm{l}$ protein $\mathrm{A} /$ $\mathrm{G}$ magnetic beads for $1 \mathrm{~h}$. After washing three times with lysis buffer $(50 \mathrm{mM}$ Tris- $\mathrm{HCl}$ [pH 7.4], $100 \mathrm{mM} \mathrm{NaCl}, 0.5 \% \mathrm{NP}-40,0.5 \%$ sodium deoxycholate, $1 / 25$ volume of Roche Protease Inhibitor Cocktail, $40 \mathrm{U} / \mathrm{ml}$ RNase inhibitor, and $20 \mu \mathrm{M}$ MG132), the immunocomplexes were analyzed by western blotting or real-time RT-PCR. For RNA pull-down with biotin-labeled DNA probes, cell lysates were incubated with biotinylated sense or antisense DNA oligomers $(1 \mu \mathrm{M}$, Supplementary Table 1) corresponding to Neat 1 for $2 \mathrm{~h}$, and then with 20 - $\mu$ streptavidincoupled agarose beads for $1 \mathrm{~h}$. After extensive washing, the precipitated complexes were analyzed by real-time RT-PCR and western blotting. For RNA pull-down with

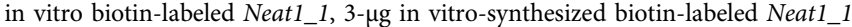
was incubated with lysates from indicated iBMDM cells $\left(4 \times 10^{7}\right)$ for $3 \mathrm{~h}$. Streptavidin-coupled agarose beads (Invitrogen) were then added to the reaction mix to isolate the RNA-protein complex. For RNA immunoprecipitation (RIP), indicated cell lysates were precleared with $20-\mu \mathrm{l}$ protein $\mathrm{A} / \mathrm{G}$ beads, and then incubated with 20 - $\mu$ protein $\mathrm{A} / \mathrm{G}$ beads coated with indicated antibodies at $4{ }^{\circ} \mathrm{C}$ for $2 \mathrm{~h}$. After extensive washing and elution, immunocomplexes were analyzed by realtime RT-PCR and western blotting. Primers used for template amplification are shown in Supplementary Table 1.

RNA fluorescence in situ hybridization following by immunofluorescence. In order to detect Neat1 and ASC in a same cell, RNA FISH was firstly carried out as previously described ${ }^{69}$ with in vitro transcribed antisense probes (Supplementary Table 1) labeled by Nucleic Acid Labeling Kits (Life technologies, USA) with Alexa Fluor 488. Then fluorescence probes-labeled cells were stained with rabbit anti-ASC and Alexa fluor 647 donkey anti-rabbit IgG as described in the next item.

Immunofluorescence. IBMDMs, seeded in glass-bottom dishes were treated with indicated conditions. The cells were fixed with $4 \%$ paraformaldehyde in PBS for 30 min, permeabilized with $0.5 \%$ Triton X-100 in PBS for $15 \mathrm{~min}$, rinsed, blocked with 5\% BSA in PBS for $30 \mathrm{~min}$, and then incubated with indicated primary antibodies overnight at $4{ }^{\circ} \mathrm{C}$. the next morning, cells were washed three times with PBST (0.05\% Tween-20 in PBS) and were recognized with indicated fluorescencelabeled secondary antibodies. After extensive washing with PBST, cells were imaging.

Pellets preparation and western blotting. Cells were harvested after LPS and nigericin co-treatment, resuspended in $0.3 \mathrm{~mL}$ buffer ( $20 \mathrm{mM}$ Hepes [pH 7.5], 150 $\mathrm{mM} \mathrm{KCl}, 1.5 \mathrm{mM} \mathrm{MgCl} 2,1 \mathrm{mM}$ EGTA, $1 \mathrm{mM}$ EDTA, $320 \mathrm{mM}$ sucrose, $1 / 25$ volume of Roche Protease Inhibitor Cocktail, $40 \mathrm{U} / \mathrm{mL}$ RNase inhibitor, $20 \mu \mathrm{M}$ MG132) lysed by shearing 10 times through a 27-gauge syringe. After centrifugation $(520 \times g, 8 \mathrm{~min})$, supernatants were mixed with equal volumes of CHAPS buffer (20 mM Hepes- $\mathrm{KOH}$ [pH 7.5], $5 \mathrm{mM} \mathrm{MgCl} 2,0.5 \mathrm{mM}$ EGTA, and $0.1 \%$ CHAPS $)$ and centrifuged $(4000 \times \mathrm{g})$ for another 8 min to pellet the ASC pyroptosomes. The pellets then were resuspended in CHAPS buffer containing 2 $\mathrm{mM}$ disuccinimidyl suberate (DSS) and incubated for $30 \mathrm{~min}$ followed by centrifugation $(4000 \times \mathrm{g}, 10 \mathrm{~min})$. Pellets were fractionated on 12\% SDS/PAGE and detected by immunoblotting with ASC antibody. Furthermore, western blotting was performed by the method described previously ${ }^{70}$.

Enzyme-linked immunosorbent assay. Supernatants from cell culture were assayed for mouse IL- $1 \beta$ and mouse TNF- $\alpha$ according to manufacturer's instructions of ELISA kits (R\&D).

Cytosolic and nuclear fractionation. Indicated cells were incubated with hypotonic buffer ( $25 \mathrm{mM}$ Tris- $\mathrm{HCl}$, $\mathrm{pH} 7.4,1 \mathrm{mM} \mathrm{MgCl}_{2}, 5 \mathrm{mM} \mathrm{KCl}$ ) on ice for $5 \mathrm{~min}$. An equal volume of hypotonic buffer containing $1 \%$ NP-40 was then added, and each sample was left on ice for another $5 \mathrm{~min}$. After centrifugation at $5000 \times g$ for 5 $\mathrm{min}$, the supernatant was collected as the cytosolic fraction. The pellets were resuspended in nucleus resuspension buffer (20 mM HEPES, pH 7.9, $400 \mathrm{mM}$ $\mathrm{NaCl}, 1 \mathrm{mM}$ EDTA, $1 \mathrm{mM}$ EGTA, $1 \mathrm{mM}$ DTT, $1 \mathrm{mM} \mathrm{PMSF}$ ) and incubated at $4{ }^{\circ} \mathrm{C}$ for $30 \mathrm{~min}$. Nuclear fraction was collected after removing insoluble membrane debris by centrifugation at $12,000 \times g$ for $10 \mathrm{~min}$.

Real-time RT-PCR. Total RNA was isolated by TRIzol reagent (Invitrogen). One microgram of RNA was used to synthesize cDNA using the RT Master Mix (Takara). Real-time PCR was performed using SYBR Green real-time PCR analysis (Takara) with the specific primers (Supplementary Table 1). PCR results, recorded as cycle threshold (Ct), were normalized against an internal control (Actin).

In vivo peritonitis. Wild-type or Neat 1 knockout mice (males, 8 weeks old) were i. p. injected with $35 \mathrm{mg} / \mathrm{kg}$ Alum for $12 \mathrm{~h}$. Peritoneal cavities were washed with $6 \mathrm{~mL}$ of PBS. The peritoneal fluids were harvested and concentrated for ELISA analysis with Amicon Ultra $10 \mathrm{~K}$ from Millipore. The concentrations of mouse IL-1 $\beta$ were measured using ELISA kits (R\&D) according to the manufacturer's instruction. PECs were analyzed by flow cytometry ${ }^{56}$.

In vivo pneumonia. Neat $1^{+/+}$or Neat $1^{-/-}$mice (males, 8 weeks old) were intranasally instilled with a mixture of flagellin $(1 \mu \mathrm{g}$ per mouse $)$ and TurboFect in vivo transfection reagent in a total volume of $50 \mu \mathrm{L}$ for $12 \mathrm{~h}$ to induce pneumonia or with isotonic saline for $12 \mathrm{~h}$ as negative control. BALF was obtained by lavaging the lung with $3 \mathrm{~mL}$ PBS containing soybean trypsin inhibitor $(100 \mu \mathrm{g} / \mathrm{mL})$. The concentrations of mouse IL- $1 \beta$ were measured using ELISA kits (R\&D). BALCs in BALF were analyzed by flow cytometry.

Flow cytometry. PECs and BALCs were obtained and blocked in PBS with $20 \%$ rat serum for $0.5 \mathrm{~h}$. After washing three times with PBS, cells were incubated with PerCP-CyTM5.5-mouse CD11b antibody, APC-mouse Ly-6G antibody, and PEmouse Ly-6C antibody for $1 \mathrm{~h}$. After washing three times with PBS, stained cells were analyzed on the BD FACSCalibur to detect the proportion of neutrophils or Ly- $6 \mathrm{C}^{+}$monocytes. The flow cytometry data were analyzed on FlowJo software with the gating strategies indicated in Supplementary Fig. 10.

shRNAs and siRNAs. Forward (F) and reverse (R) DNA fragments (Supplementary Table 1) were annealed and then cloned into plasmid pLKO.1, which was used for gene knockdown in iBMDMs. siRNAs were used for gene knockdown in BMDMs. siRNAs' target sequences are shown in Supplementary Table 1.

Reporting Summary. Further information on experimental design is available in the Nature Research Reporting Summary linked to this article.

\section{Data availability}

All relevant data are available from the corresponding authors upon reasonable request. The source data underlying all reported averages in Figs. 1b, c, e, g, i, 4a-d, 5b, d, e, f, 6b d, f, 7a-c, 8g, h, 10a-h and Supplementary Figs. 1c-i, k, l, 3b-e, 4a-h, 7a-g, 8f, 9a are provided in Source Data File. The source data underlying all uncropped versions of the blots and gels presented in Figs. 1a, d, f, h, 2a-h, 3a-d, 4a-f, 5a, c, 8a-c, e, f, 9a-i and 
Supplementary Figs. 1j, m, 2a, b, 3a, 4f, h, 5d, 6a-e, 8a, d, e, $9 \mathrm{~b}$ are also provided in Source Data File. The sequencing data were deposited in the National Center for Biotechnology Information Gene Expression Omnibus database (GSE118722).

Received: 20 September 2018 Accepted: 13 March 2019

Published online: 02 April 2019

\section{References}

1. Mariathasan, S. et al. Differential activation of the inflammasome by caspase-1 adaptors ASC and Ipaf. Nature 430, 213-218 (2004).

2. Schroder, K. \& Tschopp, J. The inflammasomes. Cell 140, 821-832 (2010).

3. Strowig, T., Henao-Mejia, J., Elinav, E. \& Flavell, R. Inflammasomes in health and disease. Nature 481, 278-286 (2012).

4. Fink, S. L. \& Cookson, B. T. Apoptosis, pyroptosis, and necrosis: mechanistic description of dead and dying eukaryotic cells. Infect. Immun. 73, 1907-1916 (2005).

5. Tang, D., Kang, R., Coyne, C. B., Zeh, H. J. \& Lotze, M. T. PAMPs and DAMPs: signal 0s that spur autophagy and immunity. Immunol. Rev. 249, 158-175 (2012).

6. Martinon, F., Mayor, A. \& Tschopp, J. The Inflammasomes: guardians of the Body. Annu. Rev. Immunol. 27, 229-265 (2009).

7. Broz, P. \& Dixit, V. M. Inflammasomes: mechanism of assembly, regulation and signalling. Nat. Rev. Immunol. 16, 407-420 (2016).

8. Thompson, M. R., Kaminski, J. J., Kurt-Jones, E. A. \& Fitzgerald, K. A. Pattern recognition receptors and the innate immune response to viral infection. Virus.-Basel 3, 920-940 (2011).

9. Kanneganti, T. D. et al. Bacterial RNA and small antiviral compounds activate caspase-1 through cryopyrin/Nalp3. Nature 440, 233-236 (2006).

10. Mariathasan, S. et al. Cryopyrin activates the inflammasome in response to toxins and ATP. Nature 440, 228-232 (2006).

11. Martinon, F., Agostini, L., Meylan, E. \& Tschopp, J. Identification of bacterial muramyl dipeptide as activator of the NALP3/cryopyrin inflammasome. Curr. Biol. 14, 1929-1934 (2004).

12. Tschopp, J. \& Schroder, K. NLRP3 inflammasome activation: the convergence of multiple signalling pathways on ROS production? Nat. Rev. Immunol. 10, 210 (2010).

13. Yu, H. B. \& Finlay, B. B. The caspase-1 inflammasome: a pilot of innate immune responses. Cell Host. Microbe 4, 198-208 (2008).

14. Ferrari, D. et al. Extracellular ATP triggers IL-1 beta release by activating the purinergic P2Z receptor of human macrophages. J. Immunol. 159, 1451-1458 (1997).

15. Munoz-Planillo, R. et al. $\mathrm{K}+$ Efflux is the common trigger of NLRP3 inflammasome activation by bacterial toxins and particulate matter. Immunity 38, 1142-1153 (2013).

16. Perregaux, D. \& Gabel, C. A. Interleukin-1-beta maturation and release in response to ATP and nigericin-evidence that potassium-depletion mediated by these agents is a necessary and common feature of their activity. J. Biol. Chem. 269, 15195-15203 (1994).

17. Marim, F. M. et al. The role of NLRP3 and AIM2 in inflammasome activation during Brucella abortus infection. Semin. Immunopathol. 39, 215-223 (2017).

18. Sharma, D. \& Kanneganti, T. D. The cell biology of inflammasomes: mechanisms of inflammasome activation and regulation. J. Cell Biol. 213, 617-629 (2016).

19. Dinarello, C. A. Anti-inflammatory agents: present and future. Cell 140, 935-950 (2010).

20. Hornung, V. et al. AIM2 recognizes cytosolic dsDNA and forms a caspase-1activating inflammasome with ASC. Nature 458, 514-U516 (2009).

21. Zhao, Y. et al. The NLRC4 inflammasome receptors for bacterial flagellin and type III secretion apparatus. Nature 477, 596-U257 (2011).

22. Boyden, E. D. \& Dietrich, W. F. Nalp1b controls mouse macrophage susceptibility to anthrax lethal toxin. Nat. Genet. 38, 240-244 (2006).

23. Liao, K. C. \& Mogridge, J. Activation of the Nlrp1b inflammasome by reduction of cytosolic ATP. Infect. Immun. 81, 570-579 (2013).

24. Gupta, N. et al. Activation of NLRP3 inflammasome complex potentiates venous thrombosis in response to hypoxia. Proc. Natl Acad. Sci. USA 114, 4763-4768 (2017).

25. Tannahill, G. M. et al. Succinate is an inflammatory signal that induces IL-1 beta through HIF-1 alpha. Nature 496, 238-+ (2013).

26. Derrien, T. et al. The GENCODE v7 catalog of human long noncoding RNAs: analysis of their gene structure, evolution, and expression. Genome Res. 22, 1775-1789 (2012).

27. Iorio, M. V. \& Croce, C. M. MicroRNA dysregulation in cancer: diagnostics, monitoring and therapeutics. a comprehensive review. EMBO Mol. Med. 4 , 143-159 (2012).
28. Mercer, T. R., Dinger, M. E. \& Mattick, J. S. Long non-coding RNAs: insights into functions. Nat. Rev. Genet. 10, 155-159 (2009).

29. Prasanth, K. V. \& Spector, D. L. Eukaryotic regulatory RNAs: an answer to the 'genome complexity' conundrum. Gene Dev. 21, 11-42 (2007).

30. Harrow, J. et al. GENCODE: the reference human genome annotation for The ENCODE Project. Genome Res. 22, 1760-1774 (2012).

31. Cabili, M. N. et al. Integrative annotation of human large intergenic noncoding RNAs reveals global properties and specific subclasses. Gene Dev. 25, 1915-1927 (2011).

32. Yi, H. et al. LincRNA-Gm4419 knockdown ameliorates NF-kappa B/NLRP3 inflammasome-mediated inflammation in diabetic nephropathy. Cell Death Dis. 8, e2583 (2017).

33. $\mathrm{Hu}, \mathrm{G}$. K. et al. LincRNA-Cox 2 promotes late inflammatory gene transcription in macrophages through modulating SWI/SNF-mediated chromatin remodeling. J. Immunol. 196, 2799-2808 (2016).

34. Atianand, M. K. et al. A long noncoding RNA lincRNA-EPS acts as a transcriptional brake to restrain inflammation. Cell 165, 1672-1685 (2016).

35. Sasaki, Y. T. F., Ideue, T., Sano, M., Mituyama, T. \& Hirose, T. MEN epsilon/ beta noncoding RNAs are essential for structural integrity of nuclear paraspeckles. Proc. Natl Acad. Sci. USA 106, 2525-2530 (2009).

36. Sunwoo, H. et al. MEN epsilon/beta nuclear-retained non-coding RNAs are up-regulated upon muscle differentiation and are essential components of paraspeckles. Genome Res. 19, 347-359 (2009).

37. Zhang, F. F. et al. Identification of the long noncoding RNA NEAT1 as a novel inflammatory regulator acting through MAPK pathway in human lupus. J. Autoimmun. 75, 96-104 (2016).

38. Martinon, F. Signaling by ROS drives inflammasome activation. Eur. J. Immunol. 40, 616-619 (2010).

39. Hwang, A. B. \& Lee, S.-J. Regulation of life span by mitochondrial respiration: the HIF-1 and ROS connection. Aging 3, 304 (2011).

40. Liu, B., Chen, Y. \& Clair, D. K. S. ROS and p53: a versatile partnership. Free Radic. Biol. Med. 44, 1529-1535 (2008).

41. Keller, M., Ruegg, A., Werner, S. \& Beer, H. D. Active caspase-1 is a regulator of unconventional protein secretion. Cell 132, 818-831 (2008).

42. Van Opdenbosch, N. et al. Activation of the NLRP1b inflammasome independently of ASC-mediated caspase-1 autoproteolysis and speck formation. Nat. Commun. 5, 3209 (2014).

43. Datta, D., McClendon, C. L., Jacobson, M. P. \& Wells, J. A. Substrate and inhibitor-induced dimerization and cooperativity in caspase-1 but not caspase-3. J. Biol. Chem. 288, 9971-9981 (2013).

44. Boucher, D. et al. Caspase-1 self-cleavage is an intrinsic mechanism to terminate inflammasome activity. J. Exp. Med. 215, 827-840 https://doi.org/ 10.1084/jem.20172222 (2018).

45. Bergsbaken, T., Fink, S. L. \& Cookson, B. T. Pyroptosis: host cell death and inflammation. Nat. Rev. Microbiol. 7, 99 (2009).

46. Hutchinson, J. N. et al. A screen for nuclear transcripts identifies two linked noncoding RNAs associated with SC35 splicing domains. BMC Genomics 8, 39 (2007).

47. Wang, Y. et al. Cellular localization of NLRP3 inflammasome. Protein Cell 4, 425-431 (2013).

48. Bakele, M. et al. Localization and functionality of the inflammasome in neutrophils. J. Biol. Chem. 289, 5320-5329 https://doi.org/10.1074/jbc. M113.505636 (2014).

49. Landeras-Bueno, S. \& Ortín, J. Regulation of influenza virus infection by long non-coding RNAs. Virus Res. 212, 78-84 (2016).

50. Wang, Z. et al. NEAT1 modulates herpes simplex virus-1 replication by regulating viral gene transcription. Cell Mol. Life Sci. 74, 1117-1131 (2017).

51. Adriaens, C. et al. p53 induces formation of NEAT1 lncRNA-containing paraspeckles that modulate replication stress response and chemosensitivity. Nat. Med. 22, 861 (2016).

52. Stegh, A. H. Targeting the p53 signaling pathway in cancer therapy-the promises, challenges and perils. Expert Opin. Ther. Tar. 16, 67-83 (2012).

53. Brady, C. A. et al. Distinct p53 transcriptional programs dictate acute DNA-damage responses and tumor suppression. Cell 145, 571-583 (2011).

54. Choudhry, H. et al. Tumor hypoxia induces nuclear paraspeckle formation through HIF-2 alpha dependent transcriptional activation of NEAT1 leading to cancer cell survival. Oncogene 34, 4482-4490 (2015).

55. Choudhry, H. \& Mole, D. R. Hypoxic regulation of the noncoding genome and NEAT1. Brief. Funct. Genom. 15, 174-185 (2016).

56. Guarda, G. et al. Type I interferon inhibits interleukin-1 production and inflammasome activation. Immunity 34, 213-223 (2011)

57. Fatica, A. \& Bozzoni, I. Long non-coding RNAs: new players in cell differentiation and development. Nat. Rev. Genet. 15, 7-21 (2014).

58. Heward, J. A. \& Lindsay, M. A. Long non-coding RNAs in the regulation of the immune response. Trends Immunol. 35, 408-419 (2014). 
59. Hung, C. L. et al. A long noncoding RNA connects c-Myc to tumor metabolism. Proc. Natl Acad. Sci. USA 111, 18697-18702 (2014)

60. Mercer, T. R. \& Mattick, J. S. Structure and function of long noncoding RNAs in epigenetic regulation. Nat. Struct. Mol. Biol. 20, 300-307 (2013).

61. Guo, H. T., Callaway, J. B. \& Ting, J. P. Y. Inflammasomes: mechanism of action, role in disease, and therapeutics. Nat. Med. 21, 677-687 (2015).

62. Duncan, J. A. \& Canna, S. W. The NLRC4 Inflammasome. Immunol. Rev. 281 115-123 (2018).

63. Lugrin, J. \& Martinon, F. The AIM2 inflammasome: Sensor of pathogens and cellular perturbations. Immunol. Rev. 281, 99-114 (2018).

64. Fernandes-Alnemri, T., Yu, J. W., Datta, P., Wu, J. H. \& Alnemri, E. S. AIM2 activates the inflammasome and cell death in response to cytoplasmic DNA. Nature 458, 509-U505 (2009).

65. Chan, D. A. \& Giaccia, A. J. Hypoxia, gene expression, and metastasis. Cancer Metast Rev. 26, 333-339 (2007).

66. Groslambert, M. \& Py, B. F. Spotlight on the NLRP3 inflammasome pathway. J. Inflamm. Res. 11, 359 (2018).

67. Huppertz, I. et al. iCLIP: Protein-RNA interactions at nucleotide resolution. Methods 65, 274-287 (2014).

68. Ule, J. et al. CLIP identifies Nova-regulated RNA networks in the brain. Science 302, 1212-1215 (2003).

69. Cao, L. M., Zhang, P. F., Li, J. M. \& Wu, M. LAST, a c-Myc-inducible long noncoding RNA, cooperates with CNBP to promote CCND1 mRNA stability in human cells. Elife 6, e30433 (2017).

70. Du, W. J. et al. TAp73 enhances the pentose phosphate pathway and supports cell proliferation. Nat. Cell Biol. 15, 991-U242 (2013).

\section{Acknowledgements}

We would like to thank Professor Zexiong Lian (South China University of Technology) for kindly providing $I L-6^{-1-}$ mice. This work was supported by grants from the National Key R\&D Program of China (2018YFA0107103 and 2016YFC1302302) and the National Natural Science Foundation of China (81430065 and 81820108021) to M.W. and the U.S. National Institutes of Health (R01CA182675 and R01CA184867) to X.Y.

\section{Author contributions}

P.Z., L.C., X.Y. and M.W. designed research; P.Z. and L.C. performed experiments and analyzed data; R.Z. helped to initiate this project and provided material support; P.Z., X.Y. and M.W. wrote and revised the manuscript.

\section{Additional information}

Supplementary Information accompanies this paper at https://doi.org/10.1038/s41467019-09482-6.

Competing interests: The authors declare no competing interests.

Reprints and permission information is available online at http://npg.nature.com/ reprintsandpermissions/

Journal peer review information: Nature Communications thanks Tsan Xiao and the other anonymous reviewer(s) for their contribution to the peer review of this work. Peer reviewer reports are available.

Publisher's note: Springer Nature remains neutral with regard to jurisdictional claims in published maps and institutional affiliations.

cc) (i) Open Access This article is licensed under a Creative Commons Attribution 4.0 International License, which permits use, sharing, adaptation, distribution and reproduction in any medium or format, as long as you give appropriate credit to the original author(s) and the source, provide a link to the Creative Commons license, and indicate if changes were made. The images or other third party material in this article are included in the article's Creative Commons license, unless indicated otherwise in a credit line to the material. If material is not included in the article's Creative Commons license and your intended use is not permitted by statutory regulation or exceeds the permitted use, you will need to obtain permission directly from the copyright holder. To view a copy of this license, visit http://creativecommons.org/ licenses/by/4.0/.

(c) The Author(s) 2019 